\title{
Transverse parton distribution and fragmentation functions at NNLO: the gluon case
}

\author{
Ming-Xing Luo, Tong-Zhi Yang, Hua Xing Zhu and Yu Jiao Zhu \\ Zhejiang Institute of Modern Physics, Department of Physics, \\ Zhejiang University, Hangzhou, 310027, China \\ E-mail: mingxingluo@zju.edu.cn, yangtz@zju.edu.cn, zhuhx@zju.edu.cn, \\ zhuyujiao@zju.edu.cn
}

ABSTRACT: We calculate in this paper the perturbative gluon transverse momentum dependent parton distribution functions (TMDPDFs) and fragmentation functions (TMDFFs) using the exponential regulator for rapidity divergences. We obtain results for both unpolarized and linearly polarized distributions through next-to-next-to leading order in strong coupling constant, and through $\mathcal{O}\left(\epsilon^{2}\right)$ in dimensional regulator. We find a nontrivial momentum conservation sum rule for the linearly polarized component for both TMDPDFs and TMDFFs in the $\mathcal{N}=1$ super-Yang-Mills theory. The TMDFFs are used to calculate the two-loop gluon jet function for the energy-energy correlator in Higgs gluonic decay in the back-to-back limit.

KEYwords: Perturbative QCD, Effective Field Theories, Resummation

ArXiv EPrint: 1909.13820 


\section{Contents}

1 Introduction 1

2 Gluon TMDPDFs 3

2.1 The bare results 4

2.2 Renormalization counter terms and zero-bin subtraction 9

2.3 Renormalized coefficient functions 9

$2.4 \mathcal{N}=1$ supersymmetry sum rule for the linearly polarized gluon contribution 12

3 Gluon TMDFFs 13

$\begin{array}{lll}3.1 & \text { Bare gluon TMDFFs from crossing } & 14\end{array}$

$\begin{array}{lll}3.2 & \text { Renormalization counter terms and zero-bin subtraction } & 16\end{array}$

$\begin{array}{lll}3.3 & \text { Renormalized coefficient functions } & 16\end{array}$

4 Gluon jet funcion for the EEC in the back-to-back limit 20

5 Conclusion

A Anomalous dimensions, splitting functions, renormalization factors and the TMD soft function

A.1 Anomalous dimensions $\quad 22$

$\begin{array}{lll}\text { A.2 Space-like splitting functions } & 23\end{array}$

A.3 Time-like splitting function 24

A.4 Renormalization factors 24

$\begin{array}{ll}\text { A.5 Renormalized TMD soft function } & 25\end{array}$

$\begin{array}{lll}\text { A.6 Bare TMD soft function } & 25\end{array}$

\section{Introduction}

Transverse Momentum Dependent Parton Distribution Functions (TMDPDFs) and Fragmentation Functions (TMDFFs) probe the transverse structure of hadrons. Analytical calculations, phenomenological applications, and experimental determinations of the TMD distributions play important role in understanding the structure of hadrons [1,2].

TMDPDFs and TMDFFs have important applications in collider processes, such as Drell-Yan [3-11] and Higgs production [12-19], top quark pair production [20-23], hadronic $J / \psi$ production, semi-inclusive deep-inelastic scattering [24-28], hadron or jet production in electron-positron annihilation [29-34], and energy correlators in both $e^{+} e^{-}$and hadron colliders $[29,35,36]$. The TMDPDFs and TMDFFs are intrinsically non-perturbative objects. However, at low transverse momentum but not yet into the non-perturbative region, the TMDPDFs and TMDFFs admit light-cone operator product expansion onto the usual collinear PDFs and FFs, with perturbative calculable matching coefficients. Knowledge for these matching coefficients at higher orders are essential for achieving precision predictions. 
In this paper, we present the results for the perturbative matching coefficients at Next-to-Next-to Leading Orders (NNLO) for gluon TMDPDFs and TMDFFs. They are relevant to the production and decay of the Higgs boson, top quark pair, and $J / \psi$ at low transverse momentum at NNLO or Nex-to-Next-to-Next-to Leading Logarithms ( ${ }^{3} \mathrm{LL}$ ) order. We also provide the bare results at NNLO through $\mathcal{O}\left(\epsilon^{2}\right)$, which do not contribute to the renormalized TMD coefficients at this order, but are relevant for future $\mathrm{N}^{3} \mathrm{LO}$ calculation. It is well-known that, direct calculation of the TMD matching coefficients requires some form of regularization in addition to the usual dimensional regularization $[1$, $5,24,37-44]$. In this paper, we adopt the exponential regularization scheme for the rapidity divergences [42].

For the gluon TMD coefficients, the non-trivial spin structure leads to two independent tensor structures in transverse impact parameter space. They are known as the unpolarized coefficients and linearly polarized coefficients [45]. The linearly polarized coefficients arise from helicity-flip contribution in gluon splitting, and are suppressed by one power of $\alpha_{s}$ compared with the unpolarized ones. Their contributions to physical cross section have been discussed for diphoton production [46], Higgs production [15, 47-49], quarkonium production [50-52], $\gamma^{*}$ plus jet production [53, 54], heavy quark pair [55] and dijet production [56]. In this paper we present results through $\mathcal{O}\left(\alpha_{s}^{2}\right)$ (two loops) for both polarizations. For the linear polarization, this is formally at the NLO accuracy, since its LO contribution already starts at $O\left(\alpha_{s}\right)$. We note that with the two-loop linearly polarized contributions presented in this paper, the linearly polarized ingredients needed for $\mathrm{N}^{3} \mathrm{LO}$ calculation $[19,57]$ for Higgs production using the $q_{T}$ subtraction formalism [58] are completed. The reason is that the pure three-loop linearly polarized component will not contribute to the cross section at $\mathcal{O}\left(\alpha_{s}^{3}\right)$, since the tree-level linear polarized contribution vanishes, and its interference with the unpolarized contribution vanishes.

As a by-product of this calculation, we find an interesting momentum conservation sum rule for the linearly polarized TMD coefficients in the $\mathcal{N}=1$ supersymmetric limit. The sum rule imposes non-trivial constraint to the gluon-to-gluon and quark-to-gluon TMD coefficients, and are found to be satisfied by our two-loop results.

The unpolarized gluon TMDPDFs have been computed before using different regulators for the rapidity divergences. Our results can be compared with them when combining with the rapidity-regulator dependent TMD soft function properly [59]. We have done this exercise and found complete agreement with refs. [60, 61]. For unpolarized gluon TMDFFs, results have also been given through NNLO [62]. Our results agree with them for most of the terms, except for a term of the form $\alpha_{s}^{2} C_{A}^{2} \pi^{4} \delta(1-z)$ in the gluon-to-gluon TMD coefficient. We have also found similar discrepancy in the quark-to-quark coefficient as reported in ref. [63]. Very recently, the two-loop results for the linearly polarized gluon TMDPDFs have been given in ref. [64]. Their results are in full agreement with ours. ${ }^{1}$

This paper is organized as follows. In section 2, we give the bare and renormalized results for the gluon TMDPDFs through NNLO. In section 3, we give the bare and renormalized results for the gluon TMDFFs through NNLO. In section 4 we use the gluon

\footnotetext{
${ }^{1}$ We thank Gutierrez-Reyes, Leal-Gomez, Scimemi, and Vladimirov for useful correspondence.
} 
TMDFFs to calculate the gluon jet function, which is relevant for the Energy-Energy Correlations for Higgs gluonic decay in the back-to-back limit. We conclude in section 5 . We collect the relevant perturbative ingredients in the appedix A.

\section{Gluon TMDPDFs}

The bare gluon TMDPDF can be defined in terms of SCET [65-69] collinear gauge fields

$$
\mathcal{B}_{g / N}^{\mathrm{bare}, \mu \nu}\left(x, b_{\perp}\right)=-x P_{+} \int \frac{d b_{-}}{4 \pi} e^{-i x b_{-} P_{+} / 2}\left\langle N(P)\left|\mathcal{A}_{n \perp}^{a, \mu}\left(0, b_{-}, b_{\perp}\right) \mathcal{A}_{n \perp}^{a, \nu}(0)\right| N(P)\right\rangle,
$$

where $A_{n \perp}^{a, \mu}$ is the gauge invariant collinear gluon field with color index $a$ and Lorentz index $\mu$. For sufficiently small $b_{\perp}$, the gluon TMDPDFs admit operator production expansion onto the usual collinear PDFs,

$$
\mathcal{B}_{g / N}^{\mathrm{bare}, \mu \nu}\left(x, b_{\perp}\right)=\sum_{i} \int_{x}^{1} \frac{d \xi}{\xi} \mathcal{I}_{g i}^{\mathrm{bare}, \mu \nu}\left(\xi, b_{\perp}\right) \phi_{i / N}^{\mathrm{bare}}(x / \xi)+\mathcal{O}\left(b_{T}^{2} \Lambda_{\mathrm{QCD}}^{2}\right)
$$

where the summation is over all parton flavors $i$. The coefficient functions can be decomposed into two independent Lorentz structures,

$$
\mathcal{I}_{g i}^{\text {bare }, \mu \nu}\left(\xi, b_{\perp}\right)=\frac{g_{\perp}^{\mu \nu}}{d-2} \mathcal{I}_{g i}^{\text {bare }}\left(\xi, b_{T}\right)+\left(\frac{g_{\perp}^{\mu \nu}}{d-2}+\frac{b_{\perp}^{\mu} b_{\perp}^{\nu}}{b_{T}^{2}}\right) \mathcal{I}_{g i}^{\text {bare }}\left(\xi, b_{T}\right)
$$

where we have defined two scalar form factor $\mathcal{I}_{\text {gi }}^{\text {bare }}$ and $\mathcal{I}_{\text {gi }}^{\prime}$ bare, which can be projected out using

$$
\begin{aligned}
\mathcal{I}_{g i}^{\text {bare }}\left(\xi, b_{T}\right) & =g_{\perp}^{\mu \nu} \mathcal{I}_{g i}^{\text {bare }, \mu \nu}\left(\xi, b_{\perp}\right), \\
\mathcal{I}_{g i}^{\text {bare }}\left(\xi, b_{T}\right) & =\frac{1}{d-3}\left[g_{\perp}^{\mu \nu}+(d-2) \frac{b_{\perp}^{\mu} b_{\perp}^{\nu}}{b_{T}^{2}}\right] \mathcal{I}_{g i}^{\text {bare }, \mu \nu}\left(\xi, b_{\perp}\right),
\end{aligned}
$$

with $b_{T}^{2}=-b_{\perp}^{2}$ and $b_{T}=\sqrt{b_{T}^{2}}$.

The matching coefficients $\mathcal{I}_{g i}^{\text {bare }, \mu \nu}\left(\xi, b_{\perp}\right)$ in eq. (2.2) do not depend on the actual hadron $N$. In actual calculation, one can replace the hadron $N$ with a partonic state $j$. Furthermore, the usual bare partonic collinear PDFs are just $\phi_{i / j}^{\text {bare }}(x)=\delta_{i j} \delta(1-x)$, so one has

$$
\mathcal{I}_{g i}^{\mathrm{bare}, \mu \nu}\left(x, b_{\perp}\right)=\mathcal{B}_{g i}^{\mathrm{bare}, \mu \nu}\left(x, b_{\perp}\right)
$$

The TMDPDFs, as well as their matching coefficients, contain both UV and rapidity divergences. We adopt dimensional regularization for the UV, and exponentional regularization [42] for the rapidity divergences. In the following subsection, we present the bare results for the coefficient functions through two loops in QCD. 


\subsection{The bare results}

The relevant diagrams for gluon TMDPDF through two loops are generated with the code QGRAF [70]. We use an in-house Mathematica code to substitute in the SCET Feynman rules, and use Form [71] to carry out necessary color and algebra manipulation. We employ reverse unitarity [72] to convert phase space integral into loop integral for the purpose of integral reduction. We use FiRE5 [73] and LiteRed [74] to reduce the integrand into the so-called master integrals by Integration-By-Parts identities [75]. The resulting master integrals have been solved in ref. [63] using differential equation method [76, 77]. For the details we refer to ref. [63].

The bare coefficient functions can be expanded in terms of QCD bare coupling as

$$
\mathcal{I}_{g i}^{\text {bare }, \mu \nu}\left(\xi, b_{\perp}, \mu, \nu, \epsilon, \alpha_{0}\right)=\sum_{k=0}^{\infty}\left(\frac{\alpha_{0} S_{\epsilon}}{4 \pi}\right)^{k} \mathcal{I}_{g i}^{(k) \text { bare }, \mu \nu}\left(\xi, b_{\perp}, \mu, \nu, \epsilon\right),
$$

and similarly for the form factor $\mathcal{I}_{g i}^{\text {bare }}$ and $\mathcal{I}_{g i}^{\text {bare }}$, where $S_{\epsilon}=\left(4 \pi e^{-\gamma_{E}} \mu_{0}^{2} / \mu^{2}\right)^{\epsilon}$. The oneloop bare coefficient functions up to $\mathcal{O}\left(\epsilon^{2}\right)$ are

$$
\begin{aligned}
\mathcal{I}_{g q}^{\prime(1) \text { bare }}= & 2 C_{F} r_{b}(x)\left(2+2 L_{\perp} \epsilon+\left(L_{\perp}^{2}+\zeta_{2}\right) \epsilon^{2}\right) \\
\mathcal{I}_{g g}^{\prime(1) \text { bare }}= & 2 C_{A} r_{b}(x)\left(2+2 L_{\perp} \epsilon+\left(L_{\perp}^{2}+\zeta_{2}\right) \epsilon^{2}\right), \\
\mathcal{I}_{g q}^{(1) \text { bare }}= & -\frac{2 C_{F} p_{g q}(x)}{\epsilon}+C_{F}\left(2 x-2 p_{g q}(x) L_{\perp}\right)+\epsilon C_{F}\left(-p_{g q}(x) L_{\perp}^{2}-\zeta_{2} p_{g q}(x)+2 x L_{\perp}\right) \\
& +\epsilon^{2} C_{F}\left(-\zeta_{2} p_{g q}(x) L_{\perp}-\frac{1}{3} p_{g q}(x) L_{\perp}^{3}-\frac{2 \zeta_{3} p_{g q}(x)}{3}+x L_{\perp}^{2}+x \zeta_{2}\right), \\
\mathcal{I}_{g g}^{(1) \text { bare }}= & \frac{C_{A}}{\epsilon^{2}}(4 \delta(1-x))+\frac{C_{A}}{\epsilon}\left(-2 \delta(1-x) L_{\nu Q}-4 p_{g g}(x)\right) \\
& +C_{A}\left\{\delta(1-x)\left(-2 L_{\perp} L_{\nu Q}-2 L_{\perp}^{2}-2 \zeta_{2}\right)-4 p_{g g}(x) L_{\perp}\right\} \\
& +\epsilon C_{A}\left\{\delta(1-x)\left(-L_{\perp}^{2} L_{\nu Q}-4 \zeta_{2} L_{\perp}-\frac{4 L_{\perp}^{3}}{3}-\zeta_{2} L_{\nu Q}-\frac{8 \zeta_{3}}{3}\right)\right. \\
& \left.+p_{g g}(x)\left(-2 L_{\perp}^{2}-2 \zeta_{2}\right)\right\}+\epsilon^{2} C_{A}\left\{\delta ( 1 - x ) \left(-\frac{1}{3} L_{\perp}^{3} L_{\nu Q}+L_{\perp}\left(-\zeta_{2} L_{\nu Q}-4 \zeta_{3}\right)\right.\right. \\
& \left.\left.-3 \zeta_{2} L_{\perp}^{2}-\frac{L_{\perp}^{4}}{2}-\frac{2}{3} \zeta_{3} L_{\nu Q}-\frac{27 \zeta_{4}}{4}\right)+p_{g g}(x)\left(-2 \zeta_{2} L_{\perp}-\frac{2 L_{\perp}^{3}}{3}-\frac{4 \zeta_{3}}{3}\right)\right\},(2.7)
\end{aligned}
$$

where we have defined

$$
r_{b}(x)=\frac{1-x}{x}, \quad L_{\nu Q}=2 L_{\nu}+L_{Q}
$$

and

$$
L_{\perp}=\ln \frac{b_{T}^{2} \mu^{2}}{b_{0}^{2}}, \quad L_{Q}=2 \ln \frac{x P_{+}}{\nu}, \quad L_{\nu}=\ln \frac{\nu^{2}}{\mu^{2}},
$$

with $b_{0}=2 e^{-\gamma_{E}}$. Our notations are the same as in ref. [63]. 
The two-loop bare results up to $\mathcal{O}\left(\epsilon^{0}\right)$ are

$$
\begin{aligned}
& \mathcal{I}_{g q}^{\prime(2) \text { bare }}=\frac{16 C_{A} C_{F}}{\epsilon^{2}} r_{b}(x)+\frac{1}{\epsilon}\left\{C_{F}^{2}\left[16 H_{1} r_{b}(x)-8 H_{0}-4(1-x)\right]-\frac{32}{3} C_{F} N_{f} T_{F} r_{b}(x)\right. \\
& \left.+C_{A} C_{F}\left[\frac{1}{3} r_{b}(x)\left(48 H_{0}+48 L_{\perp}-24 L_{\nu Q}+4(3 x+43)\right)+32 H_{0}\right]\right\} \\
& +\epsilon^{0}\left\{C _ { A } C _ { F } \left[\frac { 1 } { 3 } r _ { b } ( x ) \left(48 H_{1,1}+L_{\perp}\left(96 H_{0}-48 L_{\nu Q}+8(3 x+43)\right)-40 H_{1}\right.\right.\right. \\
& \left.\left.+48 H_{2}+\frac{8}{3}(9 x-11)-48 \zeta_{2}\right)+16 H_{0,0}+64 L_{\perp} H_{0}-\frac{8(5 x+2)}{x} H_{0}\right] \\
& +C_{F}^{2}\left[\frac{1}{3} r_{b}(x)\left(-48 H_{1,1}+96 L_{\perp} H_{1}+24 H_{1}\right)-8 H_{0,0}+\left(-16 H_{0}-8(1-x)\right) L_{\perp}\right. \\
& \left.\left.+20 H_{0}-8(1-x)\right]+\frac{1}{3} C_{F} N_{f} T_{F} r_{b}(x)\left(32 H_{1}-64 L_{\perp}-\frac{64}{3}\right)\right\}+\mathcal{O}\left(\epsilon^{1}\right), \\
& \mathcal{I}_{g g}^{\prime(2) \text { bare }}=\frac{16 C_{A}^{2}}{\epsilon^{2}} r_{b}(x)+\frac{1}{\epsilon}\left\{C_{F} N_{f} T_{F}\left(\frac{16}{3}\left(x^{2}-2 x-2\right) r_{b}(x)-16 H_{0}\right)-\frac{16}{3} C_{A} N_{f} T_{F} r_{b}(x)\right. \\
& \left.+C_{A}^{2}\left(\frac{1}{3} r_{b}(x)\left(48 H_{0}+48 H_{1}+48 L_{\perp}-24 L_{\nu Q}-4\left(2 x^{2}-4 x-45\right)\right)+32 H_{0}\right)\right\} \\
& +\epsilon^{0}\left\{C _ { A } ^ { 2 } \left[\frac { 1 } { 3 } r _ { b } ( x ) \left(L_{\perp}\left(96 H_{0}+96 H_{1}-48 L_{\nu Q}-8\left(2 x^{2}-4 x-45\right)\right)+48 H_{2}\right.\right.\right. \\
& \left.\left.-48 \zeta_{2}\right)+16 H_{0,0}+64 L_{\perp} H_{0}-\frac{4(19 x+12)}{3 x} H_{0}+\frac{4\left(11 x^{3}-39 x^{2}+62 x-37\right)}{9 x}\right] \\
& +C_{F} N_{f} T_{F}\left[-16 H_{0,0}-32 L_{\perp} H_{0}+\frac{32}{3}\left(x^{2}-2 x-2\right) L_{\perp} r_{b}(x)+\frac{16(1-x)^{3}}{x}\right] \\
& \left.+C_{A} N_{f} T_{F}\left[-\frac{16 H_{0}}{3}-\frac{32 L_{\perp} r_{b}(x)}{3}+\frac{8\left(x^{3}+3 x^{2}+16 x-17\right)}{9 x}\right]\right\}+O\left(\epsilon^{1}\right), \\
& \mathcal{I}_{g q}^{(2) \text { bare }}=\frac{-8 C_{A} C_{F}}{\epsilon^{3}} p_{g q}(x)+\frac{1}{\epsilon^{2}}\left\{C_{F}^{2}\left[-4 p_{g q}(x) H_{1}+2(2-x) H_{0}-x+4\right]\right. \\
& +\frac{8}{3} C_{F} N_{f} T_{F} p_{g q}(x)+C_{A} C_{F}\left[-4 p_{g q}(x) H_{1}+4 p_{g q}(x) L_{\nu Q}-8 p_{g q}(x) L_{\perp}\right. \\
& \left.\left.-\frac{8\left(x^{2}+x+1\right)}{x} H_{0}+\frac{2\left(4 x^{3}+4 x^{2}+46 x-53\right)}{3 x}\right]\right\} \\
& +\frac{1}{\epsilon}\left\{C_{F} N_{f} T_{F}\left[-\frac{8 p_{g q}(x)}{3} H_{1}+\frac{16 p_{g q}(x) L_{\perp}}{3}+\frac{16\left(x^{2}-5 x+5\right)}{9 x}\right]\right. \\
& +C_{F}^{2}\left[4 H_{1,1} p_{g q}(x)+2(2-x) H_{0,0}+L_{\perp}\left(-8 H_{1} p_{g q}(x)+4 H_{0}(2-x)+2(4-x)\right)\right. \\
& \left.-\frac{2\left(x^{2}-6 x+6\right)}{x} H_{1}+(-3 x-4) H_{0}+5 x+1\right]+C_{A} C_{F}\left[L _ { \perp } \left(-8 p_{g q}(x) H_{1}\right.\right. \\
& \left.+8 p_{g q}(x) L_{\nu Q}-\frac{16\left(x^{2}+x+1\right)}{x} H_{0}+\frac{4\left(4 x^{3}-2 x^{2}+46 x-53\right)}{3 x}\right)-4 x L_{\nu Q} \\
& +4 p_{g q}(-x) H_{-1,0}+4\left(-H_{1,0}-H_{1,1}-H_{2}\right) p_{g q}(x)-4(x+2) H_{0,0}-8 \zeta_{2} \\
& +\frac{2}{3}\left(8 x^{2}+15 x+36\right) H_{0}+\frac{2\left(17 x^{2}-22 x+22\right)}{3 x} H_{1}
\end{aligned}
$$




$$
\begin{aligned}
& \left.\left.-\frac{2\left(44 x^{3}-29 x^{2}+19 x+9\right)}{9 x}\right]\right\}+\epsilon^{0}\left\{C _ { A } C _ { F } \left[\frac{16}{3} L_{\perp}^{3} p_{g q}(x)+L_{\perp}^{2}\left(-8 H_{1} p_{g q}(x)\right.\right.\right. \\
& \left.+8 p_{g q}(x) L_{\nu Q}-\frac{16 H_{0}\left(x^{2}+x+1\right)}{x}+\frac{4\left(4 x^{3}-8 x^{2}+46 x-53\right)}{3 x}\right) \\
& +L_{\perp}\left(8 p_{g q}(-x) H_{-1,0}+4\left(-2 H_{1,0}-2 H_{1,1}-2 H_{2}\right) p_{g q}(x)-8(x+2) H_{0,0}\right. \\
& +\frac{4}{3}\left(8 x^{2}+15 x+36\right) H_{0}+\frac{4\left(17 x^{2}-22 x+22\right)}{3 x} H_{1}-8 x L_{\nu Q} \\
& \left.+\frac{8\left(x^{2}-4 x+2\right) \zeta_{2}}{x}-\frac{4\left(44 x^{3}-29 x^{2}+19 x+9\right)}{9 x}\right)+4 \zeta_{2} p_{g q}(x) L_{\nu Q} \\
& -4 p_{g q}(-x)\left(-2 H_{-2,0}+2 H_{-1,-1,0}-H_{-1,0,0}+H_{-1} \zeta_{2}\right) \\
& +4 p_{g q}(x)\left(H_{1,2}+H_{2,1}-H_{1,0,0}+H_{1,1,0}+H_{1,1,1}-H_{1} \zeta_{2}\right) \\
& +\frac{2}{3}\left(8 x^{2}+9 x+36\right) H_{0,0}-\frac{2\left(5 x^{2}-22 x+22\right)}{3 x} H_{1,1}+\frac{8\left(x^{2}+2\right)}{x} H_{2,0} \\
& -\frac{4\left(4 x^{3}-9 x^{2}+24 x-22\right)}{3 x} H_{1,0}+4 x H_{-1,0}-4(x+2) H_{0,0,0} \\
& -\frac{8\left(x^{2}+x+1\right) \zeta_{2}}{x} H_{0}-\frac{2}{9} H_{0}\left(88 x^{2}-6 x+249\right)+\frac{2\left(43 x^{2}-152 x+152\right)}{9 x} H_{1} \\
& +4 x H_{2}+\frac{8\left(10 x^{2}-23 x+20\right) \zeta_{3}}{3 x}-\frac{2\left(4 x^{3}-4 x^{2}+2 x+9\right) \zeta_{2}}{3 x} \\
& \left.+\frac{4\left(152 x^{3}-268 x^{2}+791 x-790\right)}{27 x}\right]+C_{F}^{2}\left[L _ { \perp } ^ { 2 } \left(-8 p_{g q}(x) H_{1}+4(2-x) H_{0}\right.\right. \\
& +2(4-x))+L_{\perp}\left(8 H_{1,1} p_{g q}(x)+4(2-x) H_{0,0}-\frac{4\left(x^{2}-6 x+6\right)}{x} H_{1}\right. \\
& \left.-2(3 x+4) H_{0}+2(5 x+1)\right)+4 p_{g q}(x)\left(-H_{1,1,1}-H_{1} \zeta_{2}\right)+\frac{2\left(x^{2}-6 x+6\right)}{x} H_{1,1} \\
& +2(2-x)\left(H_{0,0,0}+H_{0} \zeta_{2}\right)+(-3 x-4) H_{0,0}-\frac{2\left(5 x^{2}-16 x+16\right)}{x} H_{1} \\
& \left.+5(x-3) H_{0}+(4-x) \zeta_{2}-x+10\right]+C_{F} N_{f} T_{F}\left[\frac{16}{3} p_{g q}(x) L_{\perp}^{2}\right. \\
& +L_{\perp}\left(\frac{32\left(x^{2}-5 x+5\right)}{9 x}-\frac{16 p_{g q}(x)}{3} H_{1}\right)+\frac{8}{3} p_{g q}(x)\left(H_{1,1}+\zeta_{2}\right) \\
& \left.\left.-\frac{16\left(x^{2}-5 x+5\right)}{9 x} H_{1}+\frac{8\left(13 x^{2}-56 x+56\right)}{27 x}\right]\right\}+\mathcal{O}\left(\epsilon^{1}\right) \\
& \mathcal{I}_{g g}^{(2) \text { bare }}=\frac{1}{\epsilon^{4}}\left\{8 C_{A}^{2} \delta(1-x)\right\}+\frac{1}{\epsilon^{3}}\left\{\delta(1-x)\left(\frac{11 C_{A}^{2}-4 C_{A} N_{f} T_{F}}{3}-8 L_{\nu Q} C_{A}^{2}\right)\right. \\
& \left.-16 C_{A}^{2} p_{g g}(x)\right\}+\frac{1}{\epsilon^{2}}\left\{C _ { A } ^ { 2 } \left[16\left(\frac{\ln (1-x)}{1-x}\right)_{+}+8 p_{g g}(x) L_{\nu Q}-16 p_{g g}(x) L_{\perp}\right.\right. \\
& -\frac{22 p_{g g}(x)}{3}+\frac{16\left(x^{3}-x^{2}+2 x-1\right)}{x} H_{1}-\frac{8\left(x^{4}-4 x^{3}+3 x^{2}+1\right)}{(1-x) x} H_{0}
\end{aligned}
$$




$$
\begin{aligned}
& \left.-\frac{8(1-x)\left(11 x^{2}+2 x+11\right)}{3 x}\right]+\frac{8}{3} C_{A} N_{f} T_{F} p_{g g}(x) \\
& +\delta(1-x)\left[C_{A} N_{f} T_{F}\left(\frac{4 L_{\nu Q}}{3}-\frac{20}{9}\right)+C_{A}^{2}\left(-8 L_{\perp} L_{\nu Q}-8 L_{\perp}^{2}+2 L_{\nu Q}^{2}+\frac{67}{9}\right.\right. \\
& \left.\left.\left.-18 \zeta_{2}-\frac{11 L_{\nu Q}}{3}\right)\right]+C_{F} N_{f} T_{F}\left[8(x+1) H_{0}+\frac{4(1-x)\left(4 x^{2}+7 x+4\right)}{3 x}\right]\right\} \\
& +\frac{1}{\epsilon}\left\{C _ { A } ^ { 2 } \left[L _ { \perp } \left(32\left(\frac{\ln (1-x)}{1-x}\right)_{+}+16 p_{g g}(x) L_{\nu Q}+\frac{32\left(x^{3}-x^{2}+2 x-1\right)}{x} H_{1}\right.\right.\right. \\
& \left.-\frac{16\left(x^{4}-4 x^{3}+3 x^{2}+1\right)}{(1-x) x} H_{0}-\frac{16(1-x)\left(11 x^{2}+2 x+11\right)}{3 x}-\frac{44 p_{g g}(x)}{3}\right) \\
& +4 p_{g g}(-x)\left(2 H_{-1,0}+\zeta_{2}\right)-\frac{8\left(x^{2}-x-1\right)^{2}}{(1-x)(x+1)} H_{0,0}+\frac{2}{3}\left(44 x^{2}-11 x+25\right) H_{0} \\
& \left.-\frac{1}{9} p_{g g}(x)\left(72 H_{1,0}+72 H_{2}-36 \zeta_{2}+134\right)+\frac{(1-x)\left(134 x^{2}-109 x+134\right)}{9 x}\right] \\
& +C_{F} N_{f} T_{F}\left[8(x+1) H_{0,0}+\left(16(x+1) H_{0}+\frac{8(1-x)\left(4 x^{2}+7 x+4\right)}{3 x}\right) L_{\perp}\right. \\
& \left.+4(x+3) H_{0}-\frac{8(1-x)\left(x^{2}-8 x+1\right)}{3 x}\right]+C_{A} N_{f} T_{F}\left[\frac{16 p_{g g}(x) L_{\perp}}{3}+\frac{40 p_{g g}(x)}{9}\right. \\
& \left.+\frac{8}{3}(x+1) H_{0}+\frac{4(1-x)\left(13 x^{2}+4 x+13\right)}{9 x}\right]+\delta(1-x)\left[C _ { A } N _ { f } T _ { F } \left(\frac{8}{3} L_{\perp} L_{\nu Q}\right.\right. \\
& \left.+\frac{8 L_{\perp}^{2}}{3}+\frac{20 L_{\nu Q}}{9}+4 \zeta_{2}-\frac{112}{27}\right)+C_{A}^{2}\left(L_{\perp}\left(4 L_{\nu Q}^{2}-\frac{22 L_{\nu Q}}{3}-32 \zeta_{2}\right)-\frac{16 L_{\perp}^{3}}{3}\right. \\
& \left.\left.\left.-\frac{22 L_{\perp}^{2}}{3}+\left(2 \zeta_{2}-\frac{67}{9}\right) L_{\nu Q}-11 \zeta_{2}-\frac{74 \zeta_{3}}{3}+\frac{404}{27}\right)\right]\right\} \\
& +\epsilon^{0}\left\{C _ { A } ^ { 2 } \left[\frac{32}{3} p_{g g}(x) L_{\perp}^{3}+L_{\perp}^{2}\left(32\left(\frac{\ln (1-x)}{1-x}\right)_{+}+\frac{32\left(x^{3}-x^{2}+2 x-1\right)}{x} H_{1}\right.\right.\right. \\
& +16 p_{g g}(x) L_{\nu Q}-\frac{16\left(x^{4}-4 x^{3}+3 x^{2}+1\right)}{(1-x) x} H_{0}-\frac{16(1-x)\left(11 x^{2}+2 x+11\right)}{3 x} \\
& \left.-\frac{44 p_{g g}(x)}{3}\right)+L_{\perp}\left(-8 p_{g g}(-x)\left(-2 H_{-1,0}-\zeta_{2}\right)-8 p_{g g}(x)\left(2 H_{1,0}+2 H_{2}-3 \zeta_{2}\right)\right. \\
& -\frac{16\left(x^{2}-x-1\right)^{2}}{(1-x)(x+1)} H_{0,0}-\frac{268 p_{g g}(x)}{9}+\frac{4}{3}\left(44 x^{2}-11 x+25\right) H_{0} \\
& \left.+\frac{2(1-x)\left(134 x^{2}-109 x+134\right)}{9 x}\right)+8 \zeta_{2} p_{g g}(x) L_{\nu Q}+16 \zeta_{2}\left(\frac{\ln (1-x)}{1-x}\right)_{+} \\
& -8 p_{g g}(-x)\left(-2 H_{-2,0}+2 H_{-1,-1,0}-H_{-1,0,0}+H_{-1} \zeta_{2}\right) \\
& -8 p_{g g}(x)\left(-H_{1,2}-H_{2,1}+H_{1,0,0}-H_{1,1,0}-\frac{25 \zeta_{3}}{6}\right)+\frac{2}{3}\left(44 x^{2}-11 x+25\right) H_{0,0} \\
& +\frac{8(1-x)\left(11 x^{2}-x+11\right) H_{1,0}}{3 x}-\frac{1}{(1-x)(x+1)}\left(8\left(x^{2}-x-1\right)^{2} H_{0,0,0}\right.
\end{aligned}
$$




$$
\begin{aligned}
& \left.+\frac{16\left(x^{4}-x^{2}-1\right)}{x} H_{2,0}-\frac{4\left(7 x^{5}-5 x^{4}+7 x^{3}+5 x^{2}-7 x+5\right) \zeta_{3}}{x}\right)-\frac{22 \zeta_{2} p_{g g}(x)}{3} \\
& -\frac{808 p_{g g}(x)}{27}+\frac{1}{9}\left(-536 x^{2}-149 x-701\right) H_{0}+\frac{16\left(x^{3}-x^{2}+2 x-1\right) \zeta_{2}}{x} H_{1} \\
& -\frac{8\left(x^{4}-4 x^{3}+3 x^{2}+1\right) \zeta_{2}}{(1-x) x} H_{0}-\frac{2 x}{3} H_{1}+\frac{4\left(211 x^{3}-186 x^{2}+174 x-196\right)}{9 x} \\
& \left.-8(1-x) \zeta_{2}\right]+C_{A} N_{f} T_{F}\left[\frac{16}{3} p_{g g}(x) L_{\perp}^{2}+L_{\perp}\left(\frac{80 p_{g g}(x)}{9}+\frac{16}{3}(x+1) H_{0}\right.\right. \\
& \left.+\frac{8(1-x)\left(13 x^{2}+4 x+13\right)}{9 x}\right)+\frac{8}{3}(x+1) H_{0,0}+\frac{8 \zeta_{2} p_{g g}(x)}{3}+\frac{224 p_{g g}(x)}{27} \\
& \left.+\frac{4}{9}(10 x+13) H_{0}+\frac{4 x}{3} H_{1}-\frac{4\left(83 x^{3}-54 x^{2}+54 x-65\right)}{27 x}\right] \\
& +C_{F} N_{f} T_{F}\left[L_{\perp}^{2}\left(16(x+1) H_{0}+\frac{8(1-x)\left(4 x^{2}+7 x+4\right)}{3 x}\right)+L_{\perp}\left(16(x+1) H_{0,0}\right.\right. \\
& \left.+8(x+3) H_{0}-\frac{16(1-x)\left(x^{2}-8 x+1\right)}{3 x}\right)+8(x+1)\left(H_{0,0,0}+H_{0} \zeta_{2}\right) \\
& +4(x+3) H_{0,0}+24 H_{0}(x+1)+\frac{4(1-x)\left(4 x^{2}+7 x+4\right) \zeta_{2}}{3 x} \\
& \left.-\frac{8(1-x)\left(x^{2}-23 x+1\right)}{3 x}\right]+\delta(1-x)\left[C _ { A } ^ { 2 } \left(\frac{1}{9} L_{\perp}^{3}\left(48 L_{\nu Q}-88\right)\right.\right. \\
& +\frac{1}{9} L_{\perp}^{2}\left(36 L_{\nu Q}^{2}-66 L_{\nu Q}-180 \zeta_{2}-134\right) \\
& +L_{\perp}\left(\frac{1}{9}\left(108 \zeta_{2}-134\right) L_{\nu Q}-\frac{88}{3} \zeta_{2}-16 \zeta_{3}\right)+2 \zeta_{2} L_{\nu Q}^{2} \\
& \left.+\left(-\frac{11 \zeta_{2}}{3}+\frac{50 \zeta_{3}}{3}-\frac{404}{27}\right) L_{\nu Q}-\frac{67 \zeta_{2}}{3}-\frac{242 \zeta_{3}}{9}-32 \zeta_{4}+\frac{2428}{81}\right) \\
& +C_{A} N_{f} T_{F}\left(\frac{32}{9} L_{\perp}^{3}+\frac{1}{9} L_{\perp}^{2}\left(40+24 L_{\nu Q}\right)+\frac{1}{9} L_{\perp}\left(40 L_{\nu Q}+96 \zeta_{2}\right)\right. \\
& \left.\left.\left.+\frac{1}{27} L_{\nu Q}\left(112+36 \zeta_{2}\right)+\frac{20 \zeta_{2}}{3}+\frac{88 \zeta_{3}}{9}-\frac{656}{81}\right)\right]\right\}+\mathcal{O}\left(\epsilon^{1}\right)
\end{aligned}
$$

where all end point divergences $\ln ^{k}(1-x) /(1-x)$ should be taken as plus-distribution, which is defined as

$$
\int_{0}^{1} \frac{g(x)}{(1-x)_{+}}=\int_{0}^{1} \frac{g(x)-g(1)}{1-x}
$$

for a smooth test function $g(x)$. We write our results in terms of Harmonic PolyLogarithms [78]. We use the Mathematica package HpL [79] to manipulate them. We use the standard shorthand notation

$$
H_{a_{1}, \ldots, a_{n}} \equiv \operatorname{HPL}\left(a_{1}, \ldots, a_{n} ; x\right) .
$$

We have also obtained the two-loop results through $\mathcal{O}\left(\epsilon^{2}\right)$, which are required for future $\mathrm{N}^{3} \mathrm{LO}$ calculation. We do not show them here to save space, but instead include them as supplementary material. 


\subsection{Renormalization counter terms and zero-bin subtraction}

The bare results contain both UV and rapidity divergences to be renormalized, while the rapidity divergence is already renormalized within exponentional regularization upon the substitution $\tau \rightarrow 1 / \nu$. To proceed, we first perform the usual coupling constant renormalization in $\overline{\mathrm{MS}}$ scheme,

$$
\alpha_{0} \mu_{0}^{2 \epsilon}(4 \pi)^{\epsilon} e^{-\epsilon \gamma_{E}}=\alpha_{s} \mu^{2 \epsilon}\left(1-\frac{\alpha_{s}}{4 \pi} \frac{\beta_{0}}{\epsilon}+\mathcal{O}\left(\alpha_{s}^{2}\right)\right),
$$

where $\gamma_{E}=0.5772 \ldots$ is the Euler constant, $\mu_{0}$ is the mass parameter in dimensional regularization, $\alpha_{s}=\alpha_{s}(\mu)$ is the renormalized coupling evaluated at the renormalization scale $\mu$. The one-loop QCD beta function for $N_{f}$ light flavor is given by

$$
\beta_{0}=\frac{11}{3} C_{A}-\frac{4}{3} T_{F} N_{f} .
$$

Next, we perform a zero-bin subtraction [80] to remove the overlapping contributions between collinear and soft modes. For the purpose of computing perturbative matching coefficients, we can used the dimensional regularized collinear PDFs in a partonic state,

$$
\begin{aligned}
\phi_{i / j}\left(x, \alpha_{s}\right)= & \delta_{i j} \delta(1-x)-\frac{\alpha_{s}}{4 \pi} \frac{P_{i j}^{(0)}(x)}{\epsilon} \\
& +\left(\frac{\alpha_{s}}{4 \pi}\right)^{2}\left[\frac{1}{2 \epsilon^{2}}\left(\sum_{k} P_{i k}^{(0)}(x) \otimes P_{k j}^{(0)}(x)+\beta_{0} P_{i j}^{(0)}(x)\right)-\frac{P_{i j}^{(1)}(x)}{2 \epsilon}\right],
\end{aligned}
$$

where $P_{i j}(x)$ are space-like splitting kernel, whose explicit expressions are collected in the appendix. After this, the remaining UV divergences in the TMDPDFs can be removed by a multiplicative renormalization counter term. These steps can be summarized as

$$
\frac{\widetilde{\mathcal{B}}_{g / j}^{\mathrm{bare}, \mu \nu}}{\mathcal{S}_{0 \mathrm{~b}}}=Z_{g}^{B} \mathcal{B}_{g / j}^{\mu \nu}=Z_{g}^{B} \sum_{i} \mathcal{I}_{g i}^{\mu \nu} \otimes \phi_{i / j},
$$

where $\widetilde{\mathcal{B}}_{g / j}^{\text {bare } \mu \nu}$ and $\mathcal{S}_{0 \mathrm{~b}}\left(\alpha_{s}\right)$ are the bare TMDPDFs and bare zero-bin soft function in terms of renormalized coupling $\alpha_{s}, Z_{g}^{B}$ is the multiplicative operator renormalization constant, and in the last equality we use the renormalized version of operator product expansion in eq. (2.2). The zero-bin soft function can be found in appendix. A.6.

\subsection{Renormalized coefficient functions}

In this subsection, we present the detailed results for the renormalized matching coefficient functions through $\mathcal{O}\left(\alpha_{s}^{2}\right)$. The renormalized coefficients obey a RG equation

$$
\begin{aligned}
\frac{d}{d \ln \mu} \mathcal{I}_{g i}^{\mu \nu}\left(x, b_{\perp}, \mu, \nu\right)= & 2\left[\Gamma^{\mathrm{cusp}}\left(\alpha_{s}(\mu)\right) \ln \frac{\nu}{x P_{+}}+\gamma^{B}\left(\alpha_{s}(\mu)\right)\right] \mathcal{I}_{g i}^{\mu \nu}\left(x, b_{\perp}, \mu, \nu\right) \\
& -2 \sum_{j} \mathcal{I}_{g j}^{\mu \nu}\left(x, b_{\perp}, \mu, \nu\right) \otimes P_{j i}\left(x, \alpha_{s}(\mu)\right)
\end{aligned}
$$


and a rapidity evolution equation [39]

$$
\frac{d}{d \ln \nu} \mathcal{I}_{g i}^{\mu \nu}\left(x, b_{\perp}, \mu, \nu\right)=-2\left[\int_{\mu}^{b_{0} / b_{T}} \frac{d \bar{\mu}}{\bar{\mu}} \Gamma^{\mathrm{cusp}}\left(\alpha_{s}(\bar{\mu})\right)+\gamma^{R}\left(\alpha_{s}\left(b_{0} / b_{T}\right)\right)\right] \mathcal{I}_{g i}^{\mu \nu}\left(x, b_{\perp}, \mu, \nu\right) .
$$

The relevant anomalous dimensions are collected in appendix. A.1. The tensor decomposition for the renormalized quantities is the same as given in eq. (2.3). Throughout this paper, we define the pertubative expansion according to

$$
\mathcal{I}_{g i}\left(x, b_{\perp}, L_{Q}\right)=\sum_{j}\left(\frac{\alpha_{s}}{4 \pi}\right)^{j} \mathcal{I}_{g i}^{(j)}\left(x, b_{\perp}, L_{Q}\right)
$$

Then, the renormalized scalar form factors are given by

$$
\begin{aligned}
\mathcal{I}_{g i}^{\prime(1)}\left(x, b_{\perp}, L_{Q}\right)= & I_{g i}^{\prime(1)} \\
\mathcal{I}_{g i}^{\prime(2)}\left(x, b_{\perp}, L_{Q}\right)= & {\left[\left(\beta_{0}-\frac{1}{2} \Gamma_{0}^{\text {cusp }} L_{Q}+\gamma_{0}^{B}\right) I_{g i}^{\prime(1)}-\sum_{j} I_{g j}^{(1)} \otimes P_{j i}^{(0)}\right] L_{\perp} } \\
& +\gamma_{0}^{R} L_{Q} I_{g i}^{(1)}+I_{g i}^{\prime(2)}, \\
\mathcal{I}_{g i}^{(0)}\left(x, b_{\perp}, L_{Q}\right)= & \delta_{g i} \delta(1-x), \\
\mathcal{I}_{g i}^{(1)}\left(x, b_{\perp}, L_{Q}\right)= & \left(-\frac{\Gamma_{0}^{\text {cusp }}}{2} L_{\perp} L_{Q}+\gamma_{0}^{B} L_{\perp}+\gamma_{0}^{R} L_{Q}\right) \delta_{g i} \delta(1-x)-P_{g i}^{(0)}(x) L_{\perp}+I_{g i}^{(1)}(x), \\
\mathcal{I}_{g i}^{(2)}\left(x, b_{\perp}, L_{Q}\right)= & \frac{1}{8}\left(-\Gamma_{0}^{\text {cusp }} L_{Q}+2 \gamma_{0}^{B}\right)\left(-\Gamma_{0}^{\text {cusp }} L_{Q}+2 \gamma_{0}^{B}+2 \beta_{0}\right) L_{\perp}^{2} \\
& +\left(-\frac{\Gamma_{1}^{\text {cusp }}}{2} L_{Q}+\gamma_{1}^{B}+\left(-\Gamma_{0}^{\text {cusp }} L_{Q}+2 \gamma_{0}^{B}+2 \beta_{0}\right) \frac{\gamma_{0}^{R}}{2} L_{Q}\right) L_{\perp} \\
& \left.+\frac{\left(\gamma_{0}^{R}\right)^{2}}{2} L_{Q}^{2}+\gamma_{1}^{R} L_{Q}\right] \delta_{g i} \delta(1-x) \\
& +\left(\frac{1}{2} \sum_{j} P_{g j}^{(0)}(x) \otimes P_{j i}^{(0)}(x)+\frac{P_{g i}^{(0)}(x)}{2}\left(\Gamma_{0}^{\text {cusp }} L_{Q}-2 \gamma_{0}^{B}-\beta_{0}\right)\right) L_{\perp}^{2} \\
& +\left[-P_{g i}^{(1)}(x)-P_{g i}^{(0)}(x) \gamma_{0}^{R} L_{Q}-\sum_{j} I_{g j}^{(1)}(x) \otimes P_{j i}^{(0)}(x)\right. \\
& \left.+\left(-\frac{\Gamma_{0}^{\text {cusp }}}{2} L_{Q}+\gamma_{0}^{B}+\beta_{0}\right) I_{g i}^{(1)}(x)\right] L_{\perp}+\gamma_{0}^{R} L_{Q} I_{g i}^{(1)}(x)+I_{g i}^{(2)}(x),
\end{aligned}
$$

where we have shown explicitly the scale-dependent part and scale-independent part. At each order, the scale-dependent part are determined by RG equations and universal anomalous dimensions. They serve as strong check of the results from Feynman diagram calculation. The genuine new results of direct calculations are the scale-independent terms. At 
one loop they are given by

$$
\begin{aligned}
I_{g q}^{\prime(1)} & =4 C_{F} r_{b}(x), \\
I_{g g}^{\prime(1)} & =4 C_{A} r_{b}(x), \\
I_{g q}^{(1)} & =2 C_{F} x, \\
I_{g g}^{(1)} & =0,
\end{aligned}
$$

where $r_{b}(x)$ is defined in eq. (2.8). At two loops, we find

$$
\begin{aligned}
& I_{g q}^{\prime(2)}=C_{A} C_{F}\left[-16 r_{b}(x)\left(-H_{1,1}+\frac{5}{6} H_{1}-H_{2}+\zeta_{2}\right)+16 H_{0,0}-\frac{8(5 x+2)}{x} H_{0}\right. \\
& \left.+\frac{8(1-x)(9 x-11)}{9 x}\right] \\
& +C_{F}^{2}\left[-16 r_{b}(x) H_{1,1}-8 H_{0,0}+8 r_{b}(x) H_{1}+20 H_{0}-8(1-x)\right] \\
& +N_{f} C_{F} T_{F}\left[-\frac{64}{9} r_{b}(x)+\frac{32}{3} r_{b}(x) H_{1}\right] \text {, } \\
& I_{g g}^{\prime(2)}=C_{A}^{2}\left[16 r_{b}(x)\left(-\zeta_{2}+H_{2}\right)+16 H_{0,0}-\frac{4(19 x+12)}{3 x} H_{0}+\frac{4\left(11 x^{3}-39 x^{2}+62 x-37\right)}{9 x}\right] \\
& +N_{f} C_{A} T_{F}\left[\frac{8\left(x^{3}+3 x^{2}+16 x-17\right)}{9 x}-\frac{16}{3} H_{0}\right]+N_{f} C_{F} T_{F}\left[-16 H_{0,0}+\frac{16(1-x)^{3}}{x}\right], \\
& I_{g q}^{(2)}=C_{A} C_{F}\left[\frac{2}{3}\left(8 x^{2}+9 x+36\right) H_{0,0}-\frac{2\left(5 x^{2}-22 x+22\right)}{3 x} H_{1,1}+\frac{8\left(x^{2}+2\right)}{x} H_{2,0}\right. \\
& -\frac{4\left(4 x^{3}-9 x^{2}+24 x-22\right)}{3 x} H_{1,0}+4 x H_{-1,0}-4(x+2) H_{0,0,0} \\
& -\frac{2}{9}\left(88 x^{2}-6 x+249\right) H_{0}+\frac{2\left(43 x^{2}-152 x+152\right)}{9 x} H_{1}+4 x H_{2}+\frac{8\left(3 x^{2}-7 x+6\right)}{x} \zeta_{3} \\
& +\frac{8(1-x)\left(2 x^{2}-x+11\right)}{3 x} \zeta_{2}+\frac{4\left(152 x^{3}-268 x^{2}+791 x-790\right)}{27 x} \\
& +4 p_{g q}(x)\left(H_{1,2}+H_{2,1}-H_{1,0,0}+H_{1,1,0}+H_{1,1,1}\right) \\
& \left.-4 p_{g q}(-x)\left(-2 H_{-2,0}+2 H_{-1,-1,0}-H_{-1,0,0}+\zeta_{2} H_{-1}\right)\right] \\
& +C_{F}^{2}\left[\frac{2\left(x^{2}-6 x+6\right)}{x} H_{1,1}-\frac{4\left(x^{2}-2 x+2\right)}{x} H_{1,1,1}+(-3 x-4) H_{0,0}\right. \\
& \left.+2(2-x) H_{0,0,0}-\frac{2\left(5 x^{2}-16 x+16\right)}{x} H_{1}+5(x-3) H_{0}-x+10\right] \\
& +N_{f} C_{F} T_{F}\left[\frac{8\left(x^{2}-2 x+2\right)}{3 x} H_{1,1}-\frac{16\left(x^{2}-5 x+5\right)}{9 x} H_{1}+\frac{8\left(13 x^{2}-56 x+56\right)}{27 x}\right], \\
& I_{g g}^{(2)}=C_{A}^{2}\left[\left(28 \zeta_{3}-\frac{808}{27}\right) \frac{1}{(1-x)_{+}}+\frac{4\left(835 x^{3}-760 x^{2}+926 x-790\right)}{27 x}-\frac{2}{3} x H_{1}\right. \\
& +\frac{2}{3}\left(44 x^{2}-11 x+25\right) H_{0,0}-\frac{16\left(x^{4}-x^{2}-1\right)}{(1-x) x(x+1)} H_{2,0}
\end{aligned}
$$




$$
\begin{aligned}
& +\frac{1}{9}\left(-536 x^{2}-149 x-701\right) H_{0}+\frac{8(1-x)\left(11 x^{2}-x+11\right)}{3 x}\left(\zeta_{2}+H_{1,0}\right) \\
& +\frac{4\left(14 x^{5}-12 x^{4}+14 x^{3}+5 x^{2}-21 x+12\right)}{(1-x) x(x+1)} \zeta_{3}-\frac{8\left(x^{2}-x-1\right)^{2}}{(1-x)(x+1)} H_{0,0,0} \\
& -8\left(-2 H_{-2,0}+2 H_{-1,-1,0}-H_{-1,0,0}+\zeta_{2} H_{-1}\right) p_{g g}(-x) \\
& \left.-8\left(-H_{1,2}-H_{2,1}+H_{1,0,0}-H_{1,1,0}\right) p_{g g}(x)\right] \\
& +N_{f} C_{F} T_{F}\left[4(x+3) H_{0,0}+8(x+1) H_{0,0,0}+24(x+1) H_{0}-\frac{8(1-x)\left(x^{2}-23 x+1\right)}{3 x}\right] \\
& +N_{f} C_{A} T_{F}\left[\frac{224}{27} \frac{1}{(1-x)_{+}}-\frac{4\left(139 x^{3}-110 x^{2}+166 x-121\right)}{27 x}\right. \\
& \left.+\frac{8}{3}(x+1) H_{0,0}+\frac{4}{9}(10 x+13) H_{0}+\frac{4}{3} x H_{1}\right] .
\end{aligned}
$$

The two-loop scalar form factors corresponding to the first tensor structure, $I_{g i}^{(2)}$, have been computed for a while $[60,62]$. Results for the second tensor structure $I_{g i}^{\prime(2)}$, also known as the linearly polarized contribution, appeared very recently, using a different rapidity regulator [64]. Although the calculations are performed with different rapidity regulators, the results can be compared by constructing a rapidity-divergence free combination of TMDPDFs. In our case, this can be done by multiplying the renormalized coefficient functions with the square root of the TMD soft function,

$$
\widetilde{\mathcal{I}}_{g i}^{\mu \nu}\left(x, b_{\perp}, Q, \mu\right)=\mathcal{I}_{g i}^{\mu \nu}\left(x, b_{\perp}, L_{Q}\right) \sqrt{\mathcal{S}_{g g}\left(b_{\perp}, \mu, \nu\right)}
$$

for $i=q, g$, and then expand in $\alpha_{s}$. The renormalized TMD soft function can be found in ref. [59]. The explicit two-loop results for the renormalized soft function are collected in eq. (A.14) of appendix. A.5. We find that for the first tensor structure, our two-loop results are in full agreement with those in the literature [62, 81]. For the second tensor structure, we also find complete agreement with the updated version of ref. [64]. In the next subsection, we present a $\mathcal{N}=1$ supersymmetry sum-rule for the linearly polarized gluon contribution, which provides further check to our results.

\section{4 $\mathcal{N}=1$ supersymmetry sum rule for the linearly polarized gluon contri- bution}

Our explicit two-loop calculation reveals an interesting momentum conservation sum rule for the linearly polarized gluon contribution in the $\mathcal{N}=1$ supersymmetric limit. This limit is obtained from our results by setting $C_{A}=C_{F}=N_{f}$ and using $T_{F}=1 / 2$. The sum rule is then written as

$$
\left.\int_{0}^{1} d x x\left(\mathcal{I}_{g g}^{\prime}\left(x, b_{\perp}, L_{Q}\right)-\mathcal{I}_{g q}^{\prime}\left(x, b_{\perp}, L_{Q}\right)\right)\right|_{C_{F}=C_{A}=N_{f}}=0 .
$$


At one-loop, the sum rule is satisfied trivially, since $I_{q g}^{\prime(1)} / C_{F}=I_{g g}^{\prime(1)} / C_{A}=4 r_{b}(x)$, see eq. (2.21). At two loops, we find

$$
\left.\left(\mathcal{I}_{g g}^{\prime(2)}\left(x, b_{\perp}, L_{Q}\right)-\mathcal{I}_{g q}^{\prime(2)}\left(x, b_{\perp}, L_{Q}\right)\right)\right|_{C_{F}=C_{A}=N_{f}}=-\frac{8}{3 x} C_{A}^{2}\left(1+x-3 x^{2}+x^{3}+3 x \ln (x)\right),
$$

substituting this into eq. (2.24) we indeed get zero.

While it is well-known that the splitting functions obey momentum conservation sum rule, in general the sum rule breaks down for the matching coefficient functions, which are cross-section level quantities. Therefore, the sum rule in eq. (2.24) is somewhat surprising. It would be interesting to see if it continues to hold at three loops. It would also be interesting to have a structural understanding of it. Since the sum rule is nontrivial, it also provides strong check to the two-loop results for the linearly polarized gluon contribution presented in this paper.

\section{Gluon TMDFFs}

To specify the definition for the gluon TMDFFs, it is necessary to specify a reference frame first. In the hadron frame, where the detected hadron has zero transverse momentum, the gluon TMDFFs can be defined as

$$
\begin{aligned}
& \mathcal{D}_{N / g}^{\text {bare }, \mu \nu}\left(z, b_{\perp}\right) \\
& \quad=-\frac{P_{+}}{z^{2}} \sum_{X} \int \frac{d b_{-}}{4 \pi} e^{i x b_{-} P_{+} / 2}\left\langle 0\left|\mathcal{A}_{n \perp}^{a, \mu}\left(0, b_{-}, b_{\perp}\right)\right| N(P), X\right\rangle\left\langle N(P), X\left|\mathcal{A}_{n \perp}^{a, \nu}(0)\right| 0\right\rangle,
\end{aligned}
$$

where $N(P)$ has zero transverse momentum. In actual calculation, it's also convenient to define the fragmentation functions in the parton frame, where the parton which initiates the fragmentation has zero transverse momentum. The parton frame TMDFFs are related to the hadron frame ones by

$$
\mathcal{F}_{N / g}^{\text {bare }, \mu \nu}\left(z, b_{\perp} / z\right)=z^{2-2 \epsilon} \mathcal{D}_{N / g}^{\text {bare }, \mu \nu}\left(z, b_{\perp}\right),
$$

where we denote the bare TMDFFs in the parton frame by $\mathcal{F}_{N / g}^{\text {bare } \mu \nu}\left(z, b_{\perp} / z\right)$. The parton frame has the advantage that the renormalization counter terms are slightly simpler compared with the hadron frame, i.e.

$$
\begin{aligned}
\mathcal{F}_{N / g}^{\text {bare }, \mu \nu}\left(z, b_{\perp} / z, \nu\right) & =Z_{g}^{B}\left(b_{\perp}, \mu, \nu\right) \mathcal{F}_{N / g}^{\mu \nu}\left(z, b_{\perp} / z, \mu, \nu\right) \\
& =Z_{g}^{B}\left(b_{\perp}, \mu, \nu\right) \sum_{i} d_{N / i}(z, \mu) \otimes \mathcal{C}_{i g}^{\mu \nu}\left(z, b_{\perp} / z, \mu, \nu\right)+\mathcal{O}\left(b_{T}^{2} \Lambda_{\mathrm{QCD}}^{2}\right)
\end{aligned}
$$

We refer to refs. $[1,63]$ for more detailed discussion on the difference of the reference frames.

To compute the gluon TMDFFs, we can use the methods as in the calculation of TMDPDFs. Alternatively, we can exploit crossing symmetry to obtain the results from TMDPDFs. We have performed calculation in both ways, and find the same results. In the next subsection, we give some details on the calculation based on crossing relation. 


\subsection{Bare gluon TMDFFs from crossing}

To explore the crossing symmetry between the TMDPDFs and TMDFFs, we write down their definitions in momentum space. For simplicity we first consider the $g \rightarrow g$ case,

$$
\begin{aligned}
& \mathcal{B}_{g / g}^{\text {bare }, \mu \nu}\left(x, b_{\perp}, p_{1}, \nu\right) \\
& =\lim _{\tau \rightarrow 0} \frac{1}{x \bar{n} \cdot p_{1}} \int d^{d} k e^{-b_{0} \tau k^{0}+i \vec{b}_{T} \cdot \vec{k}_{T}} \delta\left(\bar{n} \cdot k-(1-x) \bar{n} \cdot p_{1}\right) \\
& \quad \times\left.\prod \int \frac{d^{d} l_{j}}{(2 \pi)^{d}} \prod \int \frac{d^{d} k_{i}}{(2 \pi)^{d}}(2 \pi) \delta_{+}\left(k_{i}^{2}\right) \delta^{(d)}\left(k-\sum_{m} k_{m}\right)\left|\mathcal{M}_{g g}^{B \mu \nu}\left(p_{1}, l_{j}, k_{i}\right)\right|^{2}\right|_{\tau=1 / \nu},
\end{aligned}
$$

for TMDPDFs. In eq. (3.4), $\left|\mathcal{M}_{g g}^{B \mu \nu}\left(p_{1}, l_{j}, k_{i}\right)\right|^{2}$ is the squared amplitudes for space-like $g \rightarrow g$ splitting, with multiple real radiations $\left(k_{i}\right)$ or virtual momentum exchange $\left(l_{j}\right)$, and $p_{1}$ is the momenta of the initial-state gluon entering hard scattering. For TMDFFs in hadron frame, we have

$$
\begin{aligned}
& \mathcal{D}_{g / g}^{\text {bare }, \mu \nu}\left(z, b_{\perp}, p_{2}, \nu\right) \\
& =\lim _{\tau \rightarrow 0} \frac{1}{\bar{n} \cdot p_{2}} \int d^{d} k e^{-b_{0} \tau k^{0}+i \vec{b}_{T} \cdot \vec{k}_{T}} \delta\left(\bar{n} \cdot k-(1 / z-1) \bar{n} \cdot p_{2}\right) \\
& \quad \times\left.\prod \int \frac{d^{d} l_{j}}{(2 \pi)^{d}} \prod \int \frac{d^{d} k_{i}}{(2 \pi)^{d}}(2 \pi) \delta_{+}\left(k_{i}^{2}\right) \delta^{(d)}\left(k-\sum_{m} k_{m}\right)\left|\mathcal{M}_{g g}^{F \mu \nu}\left(p_{2}, l_{j}, k_{i}\right)\right|^{2}\right|_{\tau=1 / \nu},
\end{aligned}
$$

where $\left|\mathcal{M}_{g g}^{F \mu \nu}\left(p_{2}, l_{j}, k_{i}\right)\right|^{2}$ is the squared amplitudes for time-like $g \rightarrow g$ splitting, and $p_{2}$ is the momenta of the final-state detected gluon, which has zero transverse momentum in hadron frame. The squared amplitudes in the integrand for TMDPDFs and TMDFFs are related through the following crossing relation,

$$
\left|\mathcal{M}_{g g}^{F \mu \nu}\left(p_{2}, l_{j}, k_{i}\right)\right|^{2}=\left|\mathcal{M}_{g g}^{B \mu \nu}\left(-p_{2}, l_{j}, k_{i}\right)\right|^{2} .
$$

It is not difficult to see that

$$
\begin{aligned}
& \mathcal{B}_{g / g}^{\text {bare }, \mu \nu}\left(\frac{1}{z}, b_{\perp},-p_{2}, \nu\right) \\
& =\lim _{\tau \rightarrow 0} \frac{z}{-\bar{n} \cdot p_{2}} \int d^{d} k e^{-b_{0} \tau k^{0}+i \vec{b}_{T} \cdot \vec{k}_{T}} \delta\left(\bar{n} \cdot k+(1-1 / z) \bar{n} \cdot p_{2}\right) \\
& \quad \times\left.\prod \int \frac{d^{d} l_{j}}{(2 \pi)^{d}} \prod \int \frac{d^{d} k_{i}}{(2 \pi)^{d}}(2 \pi) \delta_{+}\left(k_{i}^{2}\right) \delta^{(d)}\left(k-\sum_{m} k_{m}\right)\left|\mathcal{M}_{g g}^{F \mu \nu}\left(p_{2}, l_{j}, k_{i}\right)\right|^{2}\right|_{\tau=1 / \nu} \\
& =-z \mathcal{D}_{g / g}^{\text {bare }, \mu \nu}\left(z, b_{\perp}, p_{2}, \nu\right),
\end{aligned}
$$

that is,

$$
\mathcal{D}_{g / g}^{\mathrm{bare}, \mu \nu}\left(z, b_{\perp}, Q_{2}, \nu\right)=-\left.\frac{1}{z} \mathcal{B}_{g / g}^{\mathrm{bare}, \mu \nu}\left(x, b_{\perp}, Q_{1}, \nu\right)\right|_{x \rightarrow \frac{1}{z}, Q_{1} \rightarrow-Q_{2}},
$$

where we have defined

$$
Q_{1}=x \bar{n} \cdot p_{1}, \quad Q_{2}=\frac{\bar{n} \cdot p_{2}}{z}
$$


The rule $Q_{1} \rightarrow-Q_{2}$ is important for resolving the ambiguity from analytic continuation of $\ln (1-x)$ (see also discussions in [82-86] for analytic continuation of splitting funtions), which can have two possibilities,

$$
\ln (1-x) \rightarrow \ln (1-x)-\ln (x)+\kappa i \pi \quad \text { with } \quad \kappa=0 \quad \text { or } 1 .
$$

We argue that one should set $\kappa=0$ for $\ln (1-x)$ terms originate from rapidity divergences, and $\kappa=1$ for $\ln (1-x)$ terms originate from virtual corrections. For virtual corrections, the analytical continuation is unambiguous, since it is determined by Feynman's $i \varepsilon$ prescription. To understand the prescription for the $\ln (1-x)$ from rapidity divergences, we first introduce the following dimensionless variables,

$$
\begin{array}{ll}
1-x=\frac{\bar{n} \cdot k}{\bar{n} \cdot p_{1}}, & 1-y_{1}=\frac{k^{2} \bar{n} \cdot p_{1}}{2 p_{1} \cdot k \bar{n} \cdot k}, \\
\frac{1-z}{z}=\frac{\bar{n} \cdot k}{\bar{n} \cdot p_{2}}, & 1-y_{2}=\frac{k^{2} \bar{n} \cdot p_{2}}{2 p_{2} \cdot k \bar{n} \cdot k} .
\end{array}
$$

Using these variables, the exponential regulator in TMDPDFs and TMDFFs can be separately written as $[42,63]$

$$
\begin{aligned}
& \exp \left(-2 \tau k^{0}\right)=\exp \left(\frac{-\tau x k_{T}^{2}}{y_{1}(1-x) Q_{1}}-\tau(1-x) \frac{Q_{1}}{x}\right), \\
& \exp \left(-2 \tau k^{0}\right)=\exp \left(\frac{-\tau k_{T}^{2}}{y_{2}(1-z) Q_{2}}-\tau(1-z) Q_{2}\right),
\end{aligned}
$$

where we have used that $2 k^{0}=\bar{n} \cdot k+n \cdot k$. These two expressions can be exactly related to each other through the following rules,

$$
p_{1} \rightarrow-p_{2}, \quad k \rightarrow k, \quad x \rightarrow \frac{1}{z}, \quad Q_{1} \rightarrow-Q_{2} .
$$

The exponential regulator regularizes the rapidity divergences in the phase space integral only. Therefore, the $\ln (1-x)$ terms that originate from rapidity divergence should not develop imaginary part under the replacements $x \rightarrow 1 / z, Q_{1} \rightarrow-Q_{2}$. This is indeed the case since the logarithm always appear in a specific combination

$$
\ln \left(\frac{\tau x}{(1-x) Q_{1}}\right)
$$

In practical calculation, one prescription to resolve the ambiguity in eq. (3.10) is to use the the following crossing rules

$$
\begin{aligned}
\ln \left(Q_{1}\right) & \rightarrow \ln \left(Q_{2}\right)-i \pi, \\
\ln (1-x) & \rightarrow \ln (1-z)-\ln (z)+i \pi .
\end{aligned}
$$

Moreover, taking into account the relation between parton frame and hadron frame, which is given in eq. (3.2), and the color and spin factors, the analytic continuations from TMDPDFs to TMDFFs reads

$$
\begin{aligned}
& \mathcal{F}_{g / g}^{\text {bare }, \mu \nu}\left(z, b_{\perp} / z, Q_{2}, \nu\right)=-\left.z^{1-2 \epsilon} \mathcal{B}_{g / g}^{\text {bare }, \mu \nu}\left(x, b_{\perp}, Q_{1}, \nu\right)\right|_{x \rightarrow \frac{1}{z}, L_{Q_{1}} \rightarrow L_{Q_{2}}-2 i \pi} \\
& \mathcal{F}_{q / g}^{\text {bare }, \mu \nu}\left(z, b_{\perp} / z, Q_{2}, \nu\right)=\left.\frac{z^{1-2 \epsilon} T_{F}}{C_{F}(1-\epsilon)} \mathcal{B}_{g / q}^{\text {bare }, \mu \nu}\left(x, b_{\perp}, Q_{1}, \nu\right)\right|_{x \rightarrow \frac{1}{z}, L_{Q_{1}} \rightarrow L_{Q_{2}}-2 i \pi}
\end{aligned}
$$


where

$$
L_{Q_{1}}=2 \ln \left(\frac{Q_{1}}{\nu}\right), \quad L_{Q_{2}}=2 \ln \left(\frac{Q_{2}}{\nu}\right)
$$

In the final step, we take the real part in eq. (3.16). Note that the analytic continuations in eq. (3.16) are valid in the region

$$
0<x<1, \quad 0<z<1 .
$$

The contributions from the end point, $\delta(1-x)$ and $\delta(1-z)$, are invariant under crossing.

The analytic continuations in eq. (3.16) can be also used to extract time-like splitting functions from space-like ones. It is similar to the analytic continuations of splitting function performed in refs. $[84,85]$. There the calculations start from the crossing of unrenormalized structure functions and work well for two-loop splitting functions. At three loops, the direct analytic continuations cause some issues (see [84, 85] for details), so it is expected our procedure presented here may also cause similar issues at three loops. However, this is beyond the scope of this paper.

\subsection{Renormalization counter terms and zero-bin subtraction}

The renormalization of the TMDFFs are similar to the renormalization of the TMDPDFs. In the parton frame, we use the following dimensional regularized collinear FFs for the counter terms:

$$
\begin{aligned}
d_{i / j}(z, \mu)= & \delta_{i j} \delta(1-z)-\frac{\alpha_{s}(\mu)}{4 \pi} \frac{P_{i j}^{T(0)}(z)}{\epsilon} \\
& +\left(\frac{\alpha_{s}(\mu)}{4 \pi}\right)^{2}\left[\frac{1}{2 \epsilon^{2}}\left(\sum_{k} P_{i k}^{T(0)}(z) \otimes P_{k j}^{T(0)}(z)+\beta_{0} P_{i j}^{T(0)}(z)\right)-\frac{P_{i j}^{T(1)}(z)}{2 \epsilon}\right],
\end{aligned}
$$

where $P_{i j}^{T}(z)$ are the time-like splitting kernel, whose explicit expression through two loops can be found in appendix. A.3. In hadron frame, the counter terms from collinear FFs involve additional factor of $z^{-2 \epsilon}$ [62], which arises from the phase space factor. The zerobin subtraction also follows closely the TMDPDFs.

\subsection{Renormalized coefficient functions}

In this subsection, we present the renormalized coefficient functions through two loops for both polarizations. We separate the results into scale-independent part and scaledependent part. The scale-dependent part is determined by the the RG equation

$$
\begin{aligned}
\frac{d}{d \ln \mu} \mathcal{C}_{i g}^{\mu \nu}\left(z, b_{\perp} / z, \mu, \nu\right)= & 2\left[\Gamma^{\mathrm{cusp}}\left(\alpha_{s}(\mu)\right) \ln \frac{z \nu}{P_{+}}+\gamma^{B}\left(\alpha_{s}(\mu)\right)\right] \mathcal{C}_{i g}^{\mu \nu}\left(z, b_{\perp} / z, \mu, \nu\right) \\
& -2 \sum_{j} P_{i j}^{T}\left(z, \alpha_{s}(\mu)\right) \otimes \mathcal{C}_{j g}^{\mu \nu}\left(z, b_{\perp} / z, \mu, \nu\right)
\end{aligned}
$$


and rapidity evolution equation

$\frac{d}{d \ln \nu} \mathcal{C}_{i g}^{\mu \nu}\left(z, b_{\perp} / z, \mu, \nu\right)=-2\left[\int_{\mu}^{b_{0} / b_{T}} \frac{d \bar{\mu}}{\bar{\mu}} \Gamma^{\mathrm{cusp}}\left(\alpha_{s}(\bar{\mu})\right)+\gamma^{R}\left(\alpha_{s}\left(b_{0} / b_{T}\right)\right)\right] \mathcal{C}_{i g}^{\mu \nu}\left(z, b_{\perp} / z, \mu, \nu\right)$.

The two polarization form factors can be extracted through

$$
\begin{aligned}
& \mathcal{C}_{i g}\left(z, b_{\perp} / z, L_{Q}\right)=g_{\perp}^{\mu \nu} \mathcal{C}_{i g}^{\mu \nu}\left(z, b_{\perp} / z, \mu, \nu\right) \\
& \mathcal{C}_{i g}^{\prime}\left(z, b_{\perp} / z, L_{Q}\right)=\frac{1}{d-3}\left[g_{\perp}^{\mu \nu}+(d-2) \frac{b_{\perp}^{\mu} b_{\perp}^{\nu}}{b_{T}^{2}}\right] \mathcal{C}_{i g}^{\mu \nu}\left(z, b_{\perp} / z, \mu, \nu\right) .
\end{aligned}
$$

Compared with eq. (2.9), for TMDFFs we use a slightly different definition for $L_{Q}$,

$$
L_{Q}=2 \ln \frac{P_{+}}{z \nu} .
$$

The explicit solutions to both equations through to two loops are given by

$$
\begin{aligned}
\mathcal{C}_{i g}^{\prime(1)}\left(z, b_{\perp} / z, L_{Q}\right)= & C_{i g}^{\prime(1)}, \\
\mathcal{C}_{i g}^{\prime(2)}\left(z, b_{\perp} / z, L_{Q}\right)= & {\left[\left(\beta_{0}-\frac{1}{2} \Gamma_{0}^{\text {cusp }} L_{Q}+\gamma_{0}^{B}\right) C_{i g}^{\prime(1)}-\sum_{j} P_{i j}^{T(0)} \otimes C_{j g}^{\prime(1)}\right] L_{\perp} } \\
& +\gamma_{0}^{R} L_{Q} C_{i g}^{\prime(1)}+C_{i g}^{\prime(2)}, \\
\mathcal{C}_{i g}^{(0)}\left(z, b_{\perp} / z, L_{Q}\right)= & \delta_{i g} \delta(1-z), \\
\mathcal{C}_{i g}^{(1)}\left(z, b_{\perp} / z, L_{Q}\right)= & \left(-\frac{\Gamma_{0}^{\text {cusp }}}{2} L_{\perp} L_{Q}+\gamma_{0}^{B} L_{\perp}+\gamma_{0}^{R} L_{Q}\right) \delta_{i g} \delta(1-z)-P_{i g}^{T(0)}(z) L_{\perp}+C_{i g}^{(1)}(z), \\
\mathcal{C}_{i g}^{(2)}\left(z, b_{\perp} / z, L_{Q}\right)= & \frac{1}{8}\left(-\Gamma_{0}^{\text {cusp }} L_{Q}+2 \gamma_{0}^{B}\right)\left(-\Gamma_{0}^{\text {cusp }} L_{Q}+2 \gamma_{0}^{B}+2 \beta_{0}\right) L_{\perp}^{2} \\
& +\left(-\frac{\Gamma_{1}^{\text {cusp }}}{2} L_{Q}+\gamma_{1}^{B}+\left(-\Gamma_{0}^{\text {cusp }} L_{Q}+2 \gamma_{0}^{B}+2 \beta_{0}\right) \frac{\gamma_{0}^{R}}{2} L_{Q}\right) L_{\perp} \\
& \left.+\frac{\left(\gamma_{0}^{R}\right)^{2}}{2} L_{Q}^{2}+\gamma_{1}^{R} L_{Q}\right] \delta_{i g} \delta(1-z) \\
& +\left(\frac{1}{2} \sum_{j} P_{i j}^{T(0)}(z) \otimes P_{j g}^{T(0)}(z)+\frac{P_{i g}^{T(0)}(z)}{2}\left(\Gamma_{0}^{\text {cusp }} L_{Q}-2 \gamma_{0}^{B}-\beta_{0}\right)\right) L_{\perp}^{2} \\
& +\left[-P_{i g}^{T(1)}(z)-P_{i g}^{T(0)}(z) \gamma_{0}^{R} L_{Q}-\sum_{j} P_{i j}^{T(0)}(z) \otimes C_{j g}^{(1)}(z)\right. \\
& \left.+\left(-\frac{\Gamma_{0}^{\text {cusp }}}{2} L_{Q}+\gamma_{0}^{B}+\beta_{0}\right) C_{i g}^{(1)}(z)\right] L_{\perp}+\gamma_{0}^{R} L_{Q} C_{i g}^{(1)}(z)+C_{i g}^{(2)}(z) . \\
& {[(z)}
\end{aligned}
$$

Our explicit diagrammatic calculation or analytical continuation reproduces all the scaledependent terms, which serve as a first check to the calculation. The genuine new results at each order are the scale independent part. At one loop they are given by

$$
\begin{aligned}
& C_{q g}^{\prime(1)}=-4 T_{F} r_{f}(z), \\
& C_{g g}^{\prime(1)}=4 C_{A} r_{f}(z),
\end{aligned}
$$




$$
\begin{aligned}
& C_{q g}^{(1)}=4 T_{F}\left(p_{q g}(z) H_{0}+r_{f}(z)\right), \\
& C_{g g}^{(1)}=8 C_{A} p_{g g}(z) H_{0},
\end{aligned}
$$

where we have defined

$$
r_{f}(z)=(1-z) z .
$$

The two-loop results are

$$
\begin{aligned}
& C_{q g}^{\prime(2)}=C_{A} T_{F}\left[-r_{f}(z)\left(16 H_{1,0}+16 H_{1,1}-\frac{40}{3} H_{1}-32 \zeta_{2}+\frac{200}{9}\right)+16 z(2 z+3) H_{0,0}\right. \\
& \left.-\frac{8}{3}\left(17 z^{2}-26 z-3\right) H_{0}\right] \\
& +C_{F} T_{F}\left[-r_{f}(z)\left(-48 H_{1,0}-16 H_{1,1}+8 H_{1}-16 H_{2}+16 \zeta_{2}\right)-24 z(2 z-1) H_{0,0}\right. \\
& \left.-4(2-z)(2 z+1) H_{0}+4(1-z)(5 z-1)\right] \\
& +N_{f} T_{F}^{2}\left[-r_{f}(z)\left(\frac{32}{3} H_{0}+\frac{32}{3} H_{1}-\frac{64}{9}\right)\right], \\
& C_{g g}^{\prime(2)}=C_{A}^{2}\left[-r_{f}(z)\left(32 H_{1,0}+16 H_{2}+16 \zeta_{2}\right)+16(z-6) z H_{0,0}\right. \\
& \left.+\frac{4\left(12 z^{3}-67 z^{2}-12 z+4\right)}{3 z} H_{0}+\frac{4\left(99 z^{3}-80 z^{2}-15 z-1\right)}{9 z}\right] \\
& +N_{f} C_{F} T_{F}\left[48 z H_{0,0}+\frac{16\left(9 z^{2}+6 z-2\right)}{3 z} H_{0}+\frac{16(1-z)\left(28 z^{2}+10 z+1\right)}{9 z}\right] \\
& +N_{f} C_{A} T_{F}\left[\frac{8\left(17 z^{3}-16 z^{2}-3 z-1\right)}{9 z}-\frac{16}{3} z H_{0}\right], \\
& C_{q g}^{(2)}=C_{A} T_{F}\left[8 p_{q g}(-z)\left(-H_{-2,0}-H_{-1,-1,0}-\frac{3}{2} H_{-1,0,0}-\frac{1}{2} \zeta_{2} H_{-1}\right)\right. \\
& +8 p_{q g}(z)\left(H_{1,2}+H_{2,1}+H_{1,0,0}+H_{1,1,0}+\frac{1}{2} H_{1,1,1}-\frac{5}{2} \zeta_{2} H_{0}-\frac{3}{2} \zeta_{2} H_{1}-\frac{5}{2} H_{3}\right) \\
& -\frac{2}{3}\left(34 z^{2}-34 z+11\right) H_{1,1}-4\left(6 z^{2}-10 z+3\right) H_{2,0} \\
& -\frac{4\left(59 z^{3}-35 z^{2}+22 z-16\right)}{3 z} H_{0,0}-\frac{2\left(86 z^{3}-90 z^{2}+45 z-8\right)}{3 z} H_{1,0}+8(z+1) z H_{-1,0} \\
& +4(62 z+11) H_{0,0,0}+\frac{4}{9}\left(91 z^{2}-91 z+38\right) H_{1}-2\left(14 z^{2}-14 z+11\right) H_{2} \\
& +\frac{2\left(38 z^{3}-699 z^{2}-165 z+24\right)}{9 z} H_{0}+\frac{2\left(70 z^{3}-54 z^{2}+21 z+8\right)}{3 z} \zeta_{2} \\
& \left.+\frac{2\left(774 z^{3}+139 z^{2}-1223 z-148\right)}{27 z}+24 z \zeta_{3}\right] \\
& +C_{F} T_{F}\left[8 p _ { q g } ( z ) \left(-\frac{3}{2} H_{1,2}-\frac{9}{2} H_{2,0}-\frac{3}{2} H_{2,1}-\frac{9}{2} H_{1,0,0}-\frac{3}{2} H_{1,1,0}-\frac{1}{2} H_{1,1,1}\right.\right. \\
& \left.+\frac{1}{2} \zeta_{2} H_{0}+\frac{3}{2} \zeta_{2} H_{1}-\frac{1}{2} H_{3}-4 \zeta_{3}\right)+\left(68 z^{2}-52 z-7\right) H_{0,0}+6\left(10 z^{2}-10 z+3\right) H_{1,0}
\end{aligned}
$$




$$
\begin{aligned}
& +2\left(10 z^{2}-10 z+3\right) H_{1,1}-22\left(4 z^{2}-2 z+1\right) H_{0,0,0}+\left(-76 z^{2}+73 z-8\right) H_{0} \\
& -4\left(9 z^{2}-9 z+4\right) H_{1}+2\left(14 z^{2}-14 z+9\right) H_{2}+2\left(-14 z^{2}+14 z-9\right) \zeta_{2} \\
& \left.+56 z^{2}-101 z+63\right] \\
& +N_{f} T_{F}^{2}\left[8 p_{q g}(z)\left(\frac{1}{3} H_{0,0}+H_{1,0}+\frac{1}{3} H_{1,1}+H_{2}-\zeta_{2}\right)-\frac{8}{3}\left(8 z^{2}-8 z+5\right) H_{0}\right. \\
& \left.-\frac{8}{9}\left(16 z^{2}-16 z+5\right) H_{1}+\frac{32}{27}\left(17 z^{2}-17 z+7\right)\right] \text {, } \\
& C_{g g}^{(2)}=C_{A}^{2}\left[\left(28 \zeta_{3}-\frac{808}{27}\right) \frac{1}{(1-z)_{+}}-\frac{4\left(16 z^{5}-22 z^{4}+16 z^{3}+29 z^{2}-9 z+22\right)}{(1-z) z(z+1)} \zeta_{3}\right. \\
& -\frac{4\left(862 z^{3}-248 z^{2}+10 z-817\right)}{27 z}-\frac{8(1-z)\left(11 z^{2}-z+11\right)}{3 z} H_{1,0} \\
& +\frac{2\left(129 z^{3}-144 z^{2}+279 z-220\right)}{3(1-z) z} H_{0,0}-\frac{16\left(6 z^{5}-7 z^{4}+6 z^{3}+7 z^{2}-6 z+7\right)}{(1-z) z(z+1)} H_{2,0} \\
& -\frac{1072 z^{4}-407 z^{3}+726 z^{2}-1123 z-536}{9(1-z) z} H_{0}+\frac{2}{3} H_{1}-\frac{8(1-z)\left(11 z^{2}-z+11\right)}{3 z} \zeta_{2} \\
& -\frac{8\left(11 z^{5}-42 z^{4}-11 z^{3}+42 z^{2}+11 z+20\right)}{(1-z) z(z+1)} H_{0,0,0}-8 p_{g g}(-z)\left(2 H_{-2,0}+2 H_{-1,-1,0}\right. \\
& \left.\left.+3 H_{-1,0,0}+\zeta_{2} H_{-1}\right)-8 p_{g g}(z)\left(H_{1,2}+H_{2,1}+7 H_{1,0,0}+H_{1,1,0}+4 \zeta_{2} H_{0}+6 H_{3}\right)\right] \\
& +N_{f} C_{A} T_{F}\left[\frac{224}{27} \frac{1}{(1-z)_{+}}-\frac{4\left(121 z^{3}-110 z^{2}+166 z-139\right)}{27 z}\right. \\
& \left.-\frac{8\left(4 z^{4}-13 z^{3}+12 z^{2}-3 z+4\right)}{3(1-z) z} H_{0,0}-\frac{4\left(46 z^{4}-71 z^{3}+90 z^{2}-91 z+46\right)}{9(1-z) z} H_{0}-\frac{4}{3} H_{1}\right] \\
& +N_{f} C_{F} T_{F}\left[\frac{4\left(16 z^{3}+15 z^{2}+21 z+16\right)}{3 z} H_{0,0}+88(z+1) H_{0,0,0}\right. \\
& \left.-\frac{8\left(82 z^{3}+81 z^{2}+135 z-6\right)}{9 z} H_{0}-\frac{8(1-z)\left(301 z^{2}+409 z+139\right)}{27 z}\right] \text {. }
\end{aligned}
$$

We note that for the unpolarized coefficients, $C_{i g}$, the two-loop results have been given in ref. [62]. We find almost full agreement with ref. [62], except a $C_{A}^{2} \pi^{4} \delta(1-z)$ term in $C_{g g}^{(2)}$. This discrepancy is similar to a discrepancy found previously [63] for the quark TMDFFs, $C_{q q}^{(2)}$. Note that to compare with ref. [62], we have constructed the rapidity regulator independent TMDFFs, similar to eq. (2.23) for TMDPDFs. Therefore, the discrepancy is unrelated to the specific rapidity regulator employed in our calculation and in ref. [62]. Like for quark TMDFFs, we have computed the gluon jet function for the EEC in the backto-back limit and tested with known constraints to verify our results. We shall present the details of this check in the next subsection.

Our two-loop results for the linearly polarized coefficients are new. Similar to the TMDPDFs, the two-loop linearly polarized contributions involve only weight 2 functions and zeta values. Furthermore, they obey a $\mathcal{N}=1$ supersymmetric momentum-conservation 
sum rule,

$$
\left.\int_{0}^{1} d z z\left(C_{g g}^{\prime}\left(z, b_{\perp}, L_{Q}\right)+C_{q g}^{\prime}\left(z, b_{\perp}, L_{Q}\right)\right)\right|_{C_{F}=C_{A}=N_{f}}=0 .
$$

We note that there is a change of sign in the sum rule for $C_{q g}^{\prime}$ compared with the corresponding sum rule for TMDPDFs in eq. (2.24).

\section{Gluon jet funcion for the EEC in the back-to-back limit}

The gluon TMDFFs can be used to calculate the gluon jet function for the back-to-back resummation of the EEC in the Higgs gluonic decay [87]. Such jet functions also appear in the back-to-back resummation for TEEC [36]. The gluon jet function can be expanded as

$$
J^{g}\left(b_{\perp}, \mu, \nu, \alpha_{s}\right)=1+\sum_{n=1}\left(\frac{\alpha_{s}}{4 \pi}\right)^{n} J_{n}^{g}\left(b_{\perp}, \mu, \nu\right) .
$$

The expansion coefficients through two loops are given by the second moment of the gluon TMDFFs,

$$
\begin{aligned}
J_{1}^{g}= & \int_{0}^{1} d z z\left(C_{g g}^{(1)}+2 N_{f} C_{q g}^{(1)}+C_{g g}^{\prime(1)}+2 N_{f} C_{q g}^{\prime(1)}\right) \\
= & C_{A}\left(-8 \zeta_{2}-2 L_{\perp} L_{Q}+\frac{11 L_{\perp}}{3}+\frac{71}{18}\right)+N_{f}\left(-\frac{2 L_{\perp}}{3}-\frac{11}{18}\right) \\
J_{2}^{g}= & \int_{0}^{1} d z z\left(C_{g g}^{(2)}+2 N_{f} C_{q g}^{(2)}+C_{g g}^{\prime(2)}+2 N_{f} C_{q g}^{\prime(2)}\right) \\
= & C_{A} N_{f}\left(L_{\perp}\left(\frac{32 \zeta_{2}}{3}+\frac{31 L_{Q}}{9}-\frac{335}{27}\right)+L_{\perp}^{2}\left(2 L_{Q}-\frac{44}{9}\right)+\frac{56 L_{Q}}{27}\right) \\
& +C_{A}^{2}\left(L_{\perp}\left(-\frac{176 \zeta_{2}}{3}+12 \zeta_{3}+\left(20 \zeta_{2}-\frac{205}{9}\right) L_{Q}+\frac{1069}{27}\right)\right. \\
& \left.+L_{\perp}^{2}\left(2 L_{Q}^{2}-11 L_{Q}+\frac{121}{9}\right)+\left(14 \zeta_{3}-\frac{404}{27}\right) L_{Q}\right) \\
& -2 C_{F} N_{f} L_{\perp}+N_{f}^{2}\left(\frac{4}{9} L_{\perp}^{2}+\frac{22}{27} L_{\perp}\right)+c_{2}^{g},
\end{aligned}
$$

where the two-loop scale-independent constant for gluon jet is

$$
\begin{aligned}
c_{2}^{g}= & \left(\frac{109 \zeta_{2}}{9}+\frac{8 \zeta_{3}}{3}-\frac{1123}{162}\right) C_{A} N_{f}+\left(-\frac{751 \zeta_{2}}{9}-\frac{176 \zeta_{3}}{3}+135 \zeta_{4}+\frac{2590}{81}\right) C_{A}^{2} \\
& +\left(8 \zeta_{3}-\frac{37}{6}\right) C_{F} N_{f}-\frac{8 N_{f}^{2}}{81} .
\end{aligned}
$$

The gluon jet constant in eq. (4.3) enters the back-to-back contact terms in the Higgs EEC at two loops. These constants provide important ingredients for $\mathrm{N}^{3} \mathrm{LL}$ resummation of the EEC in Higgs gluonic decay in the back-to-back limit. They have also been used in ref. [88] to extract the collinear contact terms for the Higgs EEC using energy-conservation sum rule. 
We note that the two-loop jet function has also been computed explicitly [89] from taking the asymptotic expansion of the differential equation satisfied by the master integrals for the Higgs EEC at finite angle [87]. Our results in this paper for the jet function agree perfectly with the brute force calculation in ref. [89]. Another independent check of our results comes from the momentum-conservation sum rule for the EEC, discovered in refs. [90, 91]. A similar discussion for the quark jet function can be found in ref. [63].

We stress that the jet functions are the second moment of the sum of all the TMDFFs, including both the unpolarized and linearly polarized contributions. Therefore, the aforementioned checks also apply to all the TMDFFs presented in this paper.

It is also interesting to consider the contribution to gluon jet function from linearly polarized gluon alone. They are given by

$$
\begin{aligned}
J_{1}^{g, \text { l.p. }}= & \frac{C_{A}}{3}-\frac{N_{f}}{3}, \\
J_{2}^{g, \text { l.p. }}= & C_{A} N_{f}\left(\frac{8 \zeta_{2}}{3}+L_{\perp}\left(\frac{2 L_{Q}}{3}-\frac{26}{9}\right)-\frac{163}{27}\right) \\
& +C_{A}^{2}\left(-\frac{8 \zeta_{2}}{3}+L_{\perp}\left(\frac{22}{9}-\frac{2 L_{Q}}{3}\right)+\frac{107}{27}\right)+2 C_{F} N_{f}+N_{f}^{2}\left(\frac{4 L_{\perp}}{9}+\frac{2}{27}\right) .
\end{aligned}
$$

It's interesting to note that the linearly polarized gluon contribution in eq. (4.4) vanishes in the $\mathcal{N}=1$ supersymmetry limit, where $C_{F}=C_{A}=N_{f}$. This is simply the consequence of the supersymmetric momentum-conservation sum rule in eq. (3.28).

Recently, EEC in the back-to-back limit has also been studied at hadron collider, the so-called TEEC. Due to the special geometry at hadron colliders, the linearly polarized gluon does not contribute to TEEC [36]. In that case, the gluon jet function is simply given by

$$
J_{\text {TEEC }}^{g}\left(b_{\perp}, \mu, \nu, \alpha_{s}\right)=J^{g}\left(b_{\perp}, \mu, \nu, \alpha_{s}\right)-J^{g, 1 \cdot \mathrm{p} \cdot\left(b_{\perp}, \mu, \nu, \alpha_{s}\right)} .
$$

Therefore, our results also provide the missing ingredients for the $\mathrm{N}^{3} \mathrm{LL}$ resummation of the TEEC in the back-to-back limit.

\section{Conclusion}

In this paper we have presented the perturbative gluon TMD coefficient functions through two loops, for both unpolarized and linearly polarized coefficients. Our calculation was performed using the exponential regulator for the regularization of rapidity divergences. We have obtained results through $\mathcal{O}\left(\epsilon^{2}\right)$ at two loops, which are relevant for future three-loop calculation. Our results are rapidity regulator dependent. Rapidity regulator independent results can be obtained by multiplying the TMD soft function properly, see eq. (2.23). We have compared our results with those in the literatures, and found perfect agreement in most cases. However, a minor discrepancy with ref. [62] was found for the $\delta(1-z)$ term in the gluon-to-gluon TMDFF. As a by-product of this calculation, we found a momentum conservation sum rule in the $\mathcal{N}=1$ supersymmetric limit for the linear polarization of gluon TMDPDFs and TMDFFs. Our results provide important ingredients for the precision studies of gluon TMDs in collider experiments. 


\section{Acknowledgments}

We are grateful to Xing Wang, Xiaofeng Xu, Li Lin Yang for related collaborations. This work was supported in part by the National Natural Science Foundation of China under contract No. 11975200 and the Zhejiang University Fundamental Research Funds for the Central Universities (2017QNA3007, 107201*172210191).

\section{A Anomalous dimensions, splitting functions, renormalization factors and the TMD soft function}

In this appendix, we list some necessary ingredients which enter our calculation.

\section{A.1 Anomalous dimensions}

For all the anomalous dimensions entering the RGEs of various TMD functions, we define the perturbative expansion according to

$$
\gamma\left(\alpha_{s}\right)=\sum_{n=0}^{\infty}\left(\frac{\alpha_{s}}{4 \pi}\right)^{n+1} \gamma_{n},
$$

where the coefficients up to $\mathcal{O}\left(\alpha_{s}^{2}\right)$ are

$$
\begin{aligned}
\Gamma_{0}^{\text {cusp }} & =4 C_{A} \\
\Gamma_{1}^{\text {cusp }} & =C_{A}^{2}\left(\frac{268}{9}-8 \zeta_{2}\right)-\frac{80 C_{A} T_{F} N_{f}}{9}, \\
\gamma_{0}^{R} & =0 \\
\gamma_{1}^{R} & =C_{A}\left[C_{A}\left(-\frac{404}{27}+14 \zeta_{3}\right)+T_{F} N_{f} \frac{112}{27}\right] \\
\gamma_{0}^{B} & =\frac{11}{3} C_{A}-\frac{4}{3} T_{F} N_{f} \\
\gamma_{1}^{B} & =C_{A}^{2}\left(\frac{32}{3}+12 \zeta_{3}\right)+\left(-\frac{16}{3} C_{A}-4 C_{F}\right) N_{f} T_{F}, \\
\gamma_{0}^{H} & =-\frac{11}{3} C_{A}+\frac{4}{3} T_{F} N_{f}, \\
\gamma_{1}^{H} & =C_{A}^{2}\left(2 \zeta_{3}+\frac{11}{3} \zeta_{2}-\frac{692}{27}\right)+C_{A} N_{f} T_{F}\left(\frac{256}{27}-\frac{4}{3} \zeta_{2}\right)+4 C_{F} N_{f} T_{F}, \\
\gamma_{0}^{S} & =0 \\
\gamma_{1}^{S} & =C_{A}\left[C_{A}\left(-\frac{404}{27}+\frac{11}{3} \zeta_{2}+14 \zeta_{3}\right)+T_{F} N_{f}\left(\frac{112}{27}-\frac{4}{3} \zeta_{2}\right)\right]
\end{aligned}
$$

The cusp anomalous dimension $\Gamma^{\text {cusp }}$ can be found in $[92,93]$ and rapidity anomalous dimension $\gamma^{R}$ can be found in refs. [59, 94]. The hard and soft anomalous dimensions $\gamma^{H}$ and $\gamma^{S}$ can be extracted from the two-loop gluon form factor [95], and can also be found 
in, e.g., refs. [96, 97]. Finally, the beam anomalous dimension $\gamma^{B}$ is related to $\gamma^{S}$ and $\gamma^{H}$ through $\gamma^{B}=\gamma^{S}-\gamma^{H}$.

The QCD beta function is defined by

$$
\frac{d \alpha_{s}}{d \ln \mu}=\beta\left(\alpha_{s}\right)=-2 \alpha_{s} \sum_{n=0}^{\infty}\left(\frac{\alpha_{s}}{4 \pi}\right)^{n+1} \beta_{n},
$$

with $[98-102]$

$$
\begin{aligned}
& \beta_{0}=\frac{11}{3} C_{A}-\frac{4}{3} T_{F} N_{f}, \\
& \beta_{1}=\frac{34}{3} C_{A}^{2}-\frac{20}{3} C_{A} T_{F} N_{f}-4 C_{F} T_{F} N_{f},
\end{aligned}
$$

A formula particularly useful for us is

$$
\alpha_{s}\left(b_{0} / b_{T}\right)=\frac{\alpha_{s}(\mu)}{t}\left[1-\frac{\alpha_{s}(\mu)}{4 \pi} \frac{\beta_{1}}{\beta_{0}} \frac{\ln t}{t}\right]+\mathcal{O}\left(\alpha_{s}^{3}\right)
$$

where

$$
t=1-\frac{\alpha_{s}(\mu)}{4 \pi} \beta_{0} L_{\perp} .
$$

\section{A.2 Space-like splitting functions}

The LO space-like splitting functions can be written as [103]

$$
\begin{aligned}
& P_{q q}^{(0)}(z)=2 C_{F}\left[p_{q q}(z)+\frac{3}{2} \delta(1-z)\right] \\
& P_{g q}^{(0)}(z)=2 C_{F} p_{g q}(z) \\
& P_{g g}^{(0)}(z)=4 C_{A} p_{g g}(z)+\delta(1-z)\left(\frac{11}{3} C_{A}-\frac{4}{3} T_{F} N_{f}\right), \\
& P_{q g}^{(0)}(z)=2 T_{F} p_{q g}(z)
\end{aligned}
$$

where

$$
\begin{aligned}
& p_{q q}(z)=\frac{1+z^{2}}{(1-z)_{+}}, \\
& p_{g q}(z)=\frac{1+(1-z)^{2}}{z}, \\
& p_{g g}(z)=\frac{z}{(1-z)_{+}}+\frac{1-z}{z}+z(1-z), \\
& p_{q g}(z)=z^{2}+(1-z)^{2} .
\end{aligned}
$$

The NLO space-like splitting functions are [104, 105]

$$
\begin{aligned}
P_{g q}^{(1)}= & C_{A} C_{F}\left[-\frac{4}{3}\left(8 z^{2}+15 z+36\right) H_{0}+\frac{4}{3}\left(-17 z-\frac{22}{z}+22\right) H_{1}-8 p_{g q}(-z) H_{-1,0}\right. \\
& \left.+\frac{4}{9 z}\left(44 z^{3}+37 z^{2}+\left(19+36 \zeta_{2}\right) z+9\right)+8 p_{g q}(z)\left(H_{1,0}+H_{1,1}+H_{2}\right)+8(z+2) H_{0,0}\right],
\end{aligned}
$$




$$
\begin{aligned}
P_{g g}^{(1)}= & C_{A}^{2}\left[\left(\frac{268}{9}-8 \zeta_{2}\right) \frac{1}{(1-z)_{+}}+8\left(\frac{1}{1-z}-2 z^{2}+4 z+\frac{1}{1+z}\right) H_{0,0}\right. \\
& +8\left(2 z^{2}-\frac{1}{1+z}+4\right) \zeta_{2}-\frac{4}{3}\left(44 z^{2}-11 z+25\right) H_{0}+16 p_{g g}(z)\left(H_{1,0}+H_{2}\right) \\
& \left.-\frac{2}{9}(109 z+25)+\frac{4}{3}\left(9 \zeta_{3}+8\right) \delta(1-z)-16 p_{g g}(-z) H_{-1,0}\right] \\
& +N_{f} C_{F} T_{F}\left[-16(z+1) H_{0,0}-8(5 z+3) H_{0}+\frac{16}{3}\left(5 z^{2}+6 z+\frac{1}{z}-12\right)-4 \delta(1-z)\right] \\
& +N_{f} C_{A} T_{F}\left[\frac{8}{9 z}\left(23 z^{3}-19 z^{2}+29 z-23\right)-\frac{16}{3} \delta(1-z)-\frac{16(z+1)}{3} H_{0}\right] . \quad \text { (A.9) }
\end{aligned}
$$

\section{A.3 Time-like splitting function}

The LO time-like splitting functions are exactly the same as the space-like ones (the GribovLipatov reciprocity), while the NLO time-like splitting functions are given by [104, 105]

$$
\begin{aligned}
P_{q g}^{T(1)}= & C_{A} T_{F}\left[-8 p_{q g}(-z) H_{-1,0}+8 p_{q g}(z)\left(2 H_{1,0}+H_{1,1}-2 H_{2}\right)-\frac{8}{3}\left(2 z^{2}+17 z+2\right) H_{0}\right. \\
& \left.+(48 z+8) H_{0,0}-\frac{4}{3}\left(10 z^{2}-10 z+11\right) H_{1}-\frac{4}{9 z}\left(-178 z^{3}\left(95+36 \zeta_{2}\right) z^{2}-13 z+20\right)\right] \\
& +C_{F} T_{F}\left[p_{q g}(z)\left(-24 H_{1,0}-8 H_{1,1}+8 H_{2}-8 \zeta_{2}\right)+\left(-16 z^{2}+8 z-4\right) H_{0,0}\right. \\
& \left.+\left(8 z^{2}+8 z-10\right) H_{0}+\left(8 z^{2}-8 z+12\right) H_{1}-\frac{2}{3}\left(60 z^{2}-69 z+36\right)\right] \\
& +N_{f} T_{F}^{2}\left[\frac{16}{3}\left(H_{1}-H_{0}\right) p_{q g}(z)-\frac{16}{9}\left(4 z^{2}-4 z+5\right)\right], \\
P_{g g}^{T(1)}= & C_{A}^{2}\left[\left(\frac{268}{9}-8 \zeta_{2}\right) \frac{1}{(1-z)_{+}}+8\left(-\frac{3}{1-z}+2 z^{2}-8 z+\frac{1}{1+z}-\frac{4}{z}\right) H_{0,0}\right. \\
& +\frac{4}{3}\left(\frac{22}{1-z}-22 z^{2}-3 z-\frac{22}{z}-33\right) H_{0}+8\left(2 z^{2}-\frac{1}{1+z}+4\right) \zeta_{2} \\
& \left.-\frac{2}{9}(109 z+25)-16 p_{g g}(-z) H_{-1,0}+\frac{4}{3}\left(9 \zeta_{3}+8\right) \delta(1-z)-16 p_{g g}(z)\left(H_{1,0}+H_{2}\right)\right] \\
& +N_{f} C_{A} T_{F}\left[\frac{80}{9} \frac{1}{(1-z)_{+}}+\frac{16\left(2 z^{3}-3 z^{2}+3 z-2\right)}{3 z} H_{0}-\frac{16}{3} \delta(1-z)\right. \\
& \left.+\frac{8\left(23 z^{3}-19 z^{2}+29 z-23\right)}{9 z}-\frac{32}{3(1-z)} H_{0}\right] \\
& +N_{f} C_{F} T_{F}\left[16(z+1) H_{0,0}+\frac{8}{3}\left(8 z^{2}+21 z+\frac{8}{z}+15\right) H_{0}-4 \delta(1-z)\right. \\
& \left.+\frac{16}{9}\left(-41 z^{2}+27 z+\frac{23}{z}-9\right)\right] \cdot \\
& +10
\end{aligned}
$$

\section{A.4 Renormalization factors}

Similar with eq. (A.1), we define all the renormalization factors with the perturbative expansion

$$
Z\left(\alpha_{s}\right)=1+\sum_{n=1}^{\infty}\left(\frac{\alpha_{s}}{4 \pi}\right)^{n} Z_{n} .
$$


In addition to the identical renormalization factors $Z_{g}^{B}\left(b_{\perp}, \mu, \nu\right)$ for TMDPDFs and TMDFFs, the soft renormalization factor $Z_{g}^{S}\left(b_{\perp}, \mu, \nu\right)$ also enter our calculation,

$$
\mathcal{S}_{g g}^{\text {bare }}\left(b_{\perp}, \nu\right)=Z_{g}^{S}\left(b_{\perp}, \mu, \nu\right) \mathcal{S}_{g g}\left(b_{\perp}, \mu, \nu\right) .
$$

The first two orders of $Z_{g}^{B}$ and $Z_{g}^{S}$ are

$$
\begin{aligned}
Z_{1}^{B}= & \frac{1}{2 \epsilon}\left(2 \gamma_{0}^{B}-\Gamma_{0}^{\text {cusp }} L_{Q}\right), \\
Z_{2}^{B}= & \frac{1}{8 \epsilon^{2}}\left(\left(\Gamma_{0}^{\text {cusp }} L_{Q}-2 \gamma_{0}^{B}\right)^{2}+2 \beta_{0}\left(\Gamma_{0}^{\text {cusp }} L_{Q}-2 \gamma_{0}^{B}\right)\right)+\frac{1}{4 \epsilon}\left(2 \gamma_{1}^{B}-\Gamma_{1}^{\text {cusp }} L_{Q}\right), \\
Z_{1}^{S}= & \frac{1}{\epsilon^{2}} \Gamma_{0}^{\text {cusp }}+\frac{1}{\epsilon}\left(-2 \gamma_{0}^{S}-\Gamma_{0}^{\text {cusp }} L_{\nu}\right), \\
Z_{2}^{S}= & \frac{1}{2 \epsilon^{4}}\left(\Gamma_{0}^{\text {cusp }}\right)^{2}-\frac{1}{4 \epsilon^{3}}\left(\Gamma_{0}^{\text {cusp }}\left(3 \beta_{0}+8 \gamma_{0}^{S}\right)+4\left(\Gamma_{0}^{\text {cusp }}\right)^{2} L_{\nu}\right)-\frac{1}{2 \epsilon}\left(2 \gamma_{1}^{S}+\Gamma_{1}^{\text {cusp }} L_{\nu}\right) \\
& +\frac{1}{4 \epsilon^{2}}\left(\Gamma_{1}^{\text {cusp }}+2\left(2 \gamma_{0}^{S}+\Gamma_{0}^{\text {cusp }} L_{\nu}\right)\left(\beta_{0}+2 \gamma_{0}^{S}+\Gamma_{0}^{\text {cusp }} L_{\nu}\right)\right) .
\end{aligned}
$$

\section{A.5 Renormalized TMD soft function}

The exponentially regularized TMD soft function is given by [59]

$$
\mathcal{S}_{g g}\left(b_{\perp}, \mu, \nu\right)=\exp \left\{\frac{\alpha_{s}}{4 \pi}\left(c_{1}^{s}+c_{1}^{\perp}\right)+\left(\frac{\alpha_{s}}{4 \pi}\right)^{2}\left(c_{2}^{s}+c_{2}^{\perp}\right)\right\},
$$

where the scale-dependent terms are

$$
\begin{aligned}
c_{1}^{s}= & \frac{\Gamma_{0}^{\text {cusp }}}{2} L_{\perp}^{2}-L_{\perp}\left(\Gamma_{0}^{\text {cusp }} L_{R}+2 \gamma_{0}^{S}\right)+2 \gamma_{0}^{R} L_{R}, \\
c_{2}^{s}= & \frac{\beta_{0} \Gamma_{0}^{\text {cusp }}}{6} L_{\perp}^{3}+\left(\frac{\Gamma_{1}^{\text {cusp }}}{2}-\frac{\beta_{0} \Gamma_{0}^{\text {cusp }}}{2} L_{R}-\beta_{0} \gamma_{0}^{S}\right) L_{\perp}^{2} \\
& +\left(\left(2 \beta_{0} \gamma_{0}^{R}-\Gamma_{1}^{\text {cusp }}\right) L_{R}-2 \gamma_{1}^{S}+\beta_{0} c_{1}^{\perp}\right) L_{\perp}+2 \gamma_{1}^{R} L_{R},
\end{aligned}
$$

where $L_{R}=L_{\perp}+L_{\nu}$ with $L_{\nu}=\ln \left(\nu^{2} / \mu^{2}\right)$. The scale-independent terms are

$$
\begin{aligned}
& c_{1}^{\perp}=-2 C_{A} \zeta_{2}, \\
& c_{2}^{\perp}=C_{A}^{2}\left(-\frac{67}{3} \zeta_{2}-\frac{154}{9} \zeta_{3}+10 \zeta_{4}+\frac{2428}{81}\right)+C_{A} N_{f}\left(\frac{10}{3} \zeta_{2}+\frac{28}{9} \zeta_{3}-\frac{328}{81}\right) .
\end{aligned}
$$

\section{A.6 Bare TMD soft function}

The bare zero-bin soft function is equivalent to bare TMD soft function,

$$
\mathcal{S}_{0 \mathrm{~b}}\left(b_{\perp}, \nu\right)=\mathcal{S}_{g g}^{\text {bare }}\left(b_{\perp}, \nu\right)=Z_{g}^{S}\left(b_{\perp}, \mu, \nu\right)\left[\mathcal{S}_{g g}\left(b_{\perp}, \mu, \nu\right) \mid c_{1}^{s} \rightarrow c_{1}^{s^{\prime}}, c_{1}^{\perp} \rightarrow c_{1}^{\perp^{\prime}}\right],
$$

where we need to expand $c_{1}^{s}$ and $c_{1}^{\perp}$ to order $\mathcal{O}\left(\epsilon^{2}\right)$,

$$
\begin{aligned}
c_{1}^{s^{\prime}}= & c_{1}^{s}+\epsilon C_{A}\left(\frac{2 L_{\perp}^{3}}{3}-2 L_{R} L_{\perp}^{2}-2 \zeta_{2}\left(L_{\perp}+L_{R}\right)\right) \\
& +\epsilon^{2} C_{A}\left(\frac{L_{\perp}^{4}}{6}-\frac{2}{3} L_{R} L_{\perp}^{3}-\zeta_{2}\left(2 L_{\perp} L_{R}+L_{\perp}^{2}\right)-\frac{4}{3} \zeta_{3}\left(2 L_{\perp}+L_{R}\right)\right), \\
c_{1}^{\perp^{\prime}}= & c_{1}^{\perp}-C_{A}\left(\epsilon \frac{8}{3} \zeta_{3}+\epsilon^{2} \frac{27}{4} \zeta_{4}\right) .
\end{aligned}
$$


Open Access. This article is distributed under the terms of the Creative Commons Attribution License (CC-BY 4.0), which permits any use, distribution and reproduction in any medium, provided the original author(s) and source are credited.

\section{References}

[1] J. Collins, Foundations of perturbative QCD, Camb. Monogr. Part. Phys. Nucl. Phys. Cosmol. 32 (2011) 1 [INSPIRE].

[2] R. Angeles-Martinez et al., Transverse Momentum Dependent (TMD) parton distribution functions: status and prospects, Acta Phys. Polon. B 46 (2015) 2501 [arXiv:1507.05267] [INSPIRE].

[3] Y.L. Dokshitzer, D. Diakonov and S.I. Troian, On the transverse momentum distribution of massive lepton pairs, Phys. Lett. B 79 (1978) 269.

[4] G. Parisi and R. Petronzio, Small transverse momentum distributions in hard processes, Nucl. Phys. B 154 (1979) 427 [InSPIRE].

[5] J.C. Collins, D.E. Soper and G.F. Sterman, Transverse momentum distribution in Drell-Yan pair and $W$ and $Z$ boson production, Nucl. Phys. B 250 (1985) 199 [INSPIRE].

[6] P.B. Arnold and R.P. Kauffman, $W$ and $Z$ production at next-to-leading order: from large $q(t)$ to small, Nucl. Phys. B 349 (1991) 381 [inSPIRE].

[7] G.A. Ladinsky and C.P. Yuan, The nonperturbative regime in QCD resummation for gauge boson production at hadron colliders, Phys. Rev. D 50 (1994) R4239 [hep-ph/9311341] [INSPIRE].

[8] G. Bozzi et al., Production of Drell-Yan lepton pairs in hadron collisions: Transverse-momentum resummation at next-to-next-to-leading logarithmic accuracy, Phys. Lett. B 696 (2011) 207 [arXiv: 1007.2351] [INSPIRE].

[9] T. Becher, M. Neubert and D. Wilhelm, Electroweak gauge-boson production at small $q_{T}$ : infrared safety from the collinear anomaly, JHEP 02 (2012) 124 [arXiv:1109.6027] [INSPIRE].

[10] W. Bizon et al., The transverse momentum spectrum of weak gauge bosons at $N^{3} L L+$ NNLO, Eur. Phys. J. C 79 (2019) 868 [arXiv:1905.05171] [INSPIRE].

[11] V. Bertone, I. Scimemi and A. Vladimirov, Extraction of unpolarized quark transverse momentum dependent parton distributions from Drell-Yan/Z-boson production, JHEP 06 (2019) 028 [arXiv: 1902.08474] [INSPIRE].

[12] E.L. Berger and J.-w. Qiu, Differential cross-section for Higgs boson production including all orders soft gluon resummation, Phys. Rev. D 67 (2003) 034026 [hep-ph/0210135] [INSPIRE].

[13] G. Bozzi, S. Catani, D. de Florian and M. Grazzini, Transverse-momentum resummation and the spectrum of the Higgs boson at the LHC, Nucl. Phys. B 737 (2006) 73 [hep-ph/0508068] [INSPIRE].

[14] Y. Gao, C.S. Li and J.J. Liu, Transverse momentum resummation for Higgs production in soft-collinear effective theory, Phys. Rev. D 72 (2005) 114020 [hep-ph/0501229] [INSPIRE].

[15] M.G. Echevarria, T. Kasemets, P.J. Mulders and C. Pisano, QCD evolution of (un)polarized gluon TMDPDFs and the Higgs $q_{T}$-distribution, JHEP 07 (2015) 158 [Erratum ibid. 05 (2017) 073] [arXiv: 1502.05354] [INSPIRE]. 
[16] D. Neill, I.Z. Rothstein and V. Vaidya, The Higgs transverse momentum distribution at NNLL and its theoretical errors, JHEP 12 (2015) 097 [arXiv:1503.00005] [INSPIRE].

[17] W. Bizon et al., Momentum-space resummation for transverse observables and the Higgs $p_{\perp}$ at $N^{3} L L+N N L O, J H E P 02$ (2018) 108 [arXiv:1705.09127] [INSPIRE].

[18] X. Chen et al., Precise QCD description of the Higgs boson transverse momentum spectrum, Phys. Lett. B 788 (2019) 425 [arXiv: 1805.00736] [INSPIRE].

[19] W. Bizoń et al., Fiducial distributions in Higgs and Drell-Yan production at $N^{3} L L+N N L O$, JHEP 12 (2018) 132 [arXiv:1805.05916] [INSPIRE].

[20] H.X. Zhu et al., Transverse-momentum resummation for top-quark pairs at hadron colliders, Phys. Rev. Lett. 110 (2013) 082001 [arXiv:1208.5774] [InSPIRE].

[21] H.T. Li et al., Top quark pair production at small transverse momentum in hadronic collisions, Phys. Rev. D 88 (2013) 074004 [arXiv: 1307.2464] [INSPIRE].

[22] S. Catani, M. Grazzini and A. Torre, Transverse-momentum resummation for heavy-quark hadroproduction, Nucl. Phys. B 890 (2014) 518 [arXiv:1408.4564] [INSPIRE].

[23] S. Catani, M. Grazzini and H. Sargsyan, Transverse-momentum resummation for top-quark pair production at the LHC, JHEP 11 (2018) 061 [arXiv:1806.01601] [INSPIRE].

[24] X.-d. Ji, J.-p. Ma and F. Yuan, QCD factorization for semi-inclusive deep-inelastic scattering at low transverse momentum, Phys. Rev. D 71 (2005) 034005 [hep-ph/0404183] [INSPIRE].

[25] X.-d. Ji, J.-P. Ma and F. Yuan, QCD factorization for spin-dependent cross sections in DIS and Drell-Yan processes at low transverse momentum, Phys. Lett. B 597 (2004) 299 [hep-ph/0405085] [INSPIRE].

[26] P. Sun, J. Isaacson, C.P. Yuan and F. Yuan, Nonperturbative functions for SIDIS and Drell-Yan processes, Int. J. Mod. Phys. A 33 (2018) 1841006 [arXiv:1406.3073] [InSPIRE].

[27] Z.-B. Kang, A. Prokudin, P. Sun and F. Yuan, Extraction of quark transversity distribution and collins fragmentation functions with QCD evolution, Phys. Rev. D 93 (2016) 014009 [arXiv: 1505. 05589] [INSPIRE].

[28] X. Liu, F. Ringer, W. Vogelsang and F. Yuan, Lepton-jet correlations in deep inelastic scattering at the electron-ion collider, Phys. Rev. Lett. 122 (2019) 192003 [arXiv: 1812.08077] [INSPIRE].

[29] J.C. Collins and D.E. Soper, Back-to-back jets in QCD, Nucl. Phys. B 193 (1981) 381 [Erratum ibid. B 213 (1983) 545] [INSPIRE].

[30] J.C. Collins and D.E. Soper, Back-to-back jets: Fourier transform from B to K-transverse, Nucl. Phys. B 197 (1982) 446 [InSPIRE].

[31] D. Neill, I. Scimemi and W.J. Waalewijn, Jet axes and universal transverse-momentum-dependent fragmentation, JHEP 04 (2017) 020 [arXiv:1612.04817] [INSPIRE].

[32] D. Gutierrez-Reyes, I. Scimemi, W.J. Waalewijn and L. Zoppi, Transverse momentum dependent distributions with jets, Phys. Rev. Lett. 121 (2018) 162001 [arXiv:1807.07573] [INSPIRE].

[33] D. Gutierrez-Reyes, I. Scimemi, W.J. Waalewijn and L. Zoppi, Transverse momentum dependent distributions in $e^{+} e^{-}$and semi-inclusive deep-inelastic scattering using jets, JHEP 10 (2019) 031 [arXiv:1904.04259] [INSPIRE]. 
[34] D. Gutierrez-Reyes et al., Probing Transverse-Momentum Distributions With Groomed Jets, JHEP 08 (2019) 161 [arXiv:1907.05896] [INSPIRE].

[35] I. Moult and H.X. Zhu, Simplicity from recoil: the three-loop soft function and factorization for the energy-energy correlation, JHEP 08 (2018) 160 [arXiv: 1801.02627] [INSPIRE].

[36] A. Gao, H.T. Li, I. Moult and H.X. Zhu, Precision QCD event shapes at hadron colliders: the transverse energy-energy correlator in the back-to-back limit, Phys. Rev. Lett. 123 (2019) 062001 [arXiv: 1901.04497] [inSPIRE].

[37] T. Becher and M. Neubert, Drell-Yan production at small $q_{T}$, transverse parton distributions and the collinear anomaly, Eur. Phys. J. C 71 (2011) 1665 [arXiv: 1007.4005] [INSPIRE].

[38] T. Becher and G. Bell, Analytic regularization in soft-collinear effective theory, Phys. Lett. B 713 (2012) 41 [arXiv:1112.3907] [INSPIRE].

[39] J.-Y. Chiu, A. Jain, D. Neill and I.Z. Rothstein, A formalism for the systematic treatment of rapidity logarithms in quantum field theory, JHEP 05 (2012) 084 [arXiv:1202.0814] [INSPIRE].

[40] J.-y. Chiu et al., Soft-collinear factorization and zero-bin subtractions, Phys. Rev. D 79 (2009) 053007 [arXiv:0901.1332] [inSPIRE].

[41] M.G. Echevarria, I. Scimemi and A. Vladimirov, Universal transverse momentum dependent soft function at NNLO, Phys. Rev. D 93 (2016) 054004 [arXiv:1511.05590] [INSPIRE].

[42] Y. Li, D. Neill and H.X. Zhu, An exponential regulator for rapidity divergences, arXiv: 1604.00392 [inSPIRE].

[43] M.A. Ebert et al., Subleading power rapidity divergences and power corrections for $q_{T}$, JHEP 04 (2019) 123 [arXiv:1812.08189] [INSPIRE].

[44] H.-Y. Liu, Y.-Q. Ma and K.-T. Chao, Improvement for color glass condensate factorization: single hadron production in pA collisions at next-to-leading order, Phys. Rev. D 100 (2019) 071503 [arXiv: 1909.02370] [INSPIRE].

[45] P.J. Mulders and J. Rodrigues, Transverse momentum dependence in gluon distribution and fragmentation functions, Phys. Rev. D 63 (2001) 094021 [hep-ph/0009343] [InSPIRE].

[46] P.M. Nadolsky, C. Balázs, E.L. Berger and C.P. Yuan, Gluon-gluon contributions to the production of continuum diphoton pairs at hadron colliders, Phys. Rev. D 76 (2007) 013008 [hep-ph/0702003] [INSPIRE].

[47] S. Catani and M. Grazzini, QCD transverse-momentum resummation in gluon fusion processes, Nucl. Phys. B 845 (2011) 297 [arXiv:1011.3918] [INSPIRE].

[48] D. Boer et al., Linearly polarized gluons and the Higgs transverse momentum distribution, Phys. Rev. Lett. 108 (2012) 032002 [arXiv:1109.1444] [InSPIRE].

[49] D. Boer, W.J. den Dunnen, C. Pisano and M. Schlegel, Determining the Higgs spin and parity in the diphoton decay channel, Phys. Rev. Lett. 111 (2013) 032002 [arXiv: 1304.2654] [INSPIRE].

[50] W.J. den Dunnen, J.P. Lansberg, C. Pisano and M. Schlegel, Accessing the transverse dynamics and polarization of gluons inside the proton at the LHC, Phys. Rev. Lett. 112 (2014) 212001 [arXiv:1401.7611] [INSPIRE].

[51] A. Mukherjee and S. Rajesh, Linearly polarized gluons in charmonium and bottomonium production in color octet model, Phys. Rev. D 95 (2017) 034039 [arXiv:1611.05974] [INSPIRE]. 
[52] J.-P. Lansberg, C. Pisano, F. Scarpa and M. Schlegel, Pinning down the linearly-polarised gluons inside unpolarised protons using quarkonium-pair production at the LHC, Phys. Lett. B 784 (2018) 217 [Erratum ibid. B 791 (2019) 420] [arXiv: 1710.01684] [INSPIRE].

[53] F. Dominguez, J.-W. Qiu, B.-W. Xiao and F. Yuan, On the linearly polarized gluon distributions in the color dipole model, Phys. Rev. D 85 (2012) 045003 [arXiv:1109.6293] [INSPIRE].

[54] D. Boer, P.J. Mulders, J. Zhou and Y.-j. Zhou, Suppression of maximal linear gluon polarization in angular asymmetries, JHEP 10 (2017) 196 [arXiv:1702.08195] [INSPIRE].

[55] C. Pisano et al., Linear polarization of gluons and photons in unpolarized collider experiments, JHEP 10 (2013) 024 [arXiv:1307.3417] [INSPIRE].

[56] D. Boer, P.J. Mulders, C. Pisano and J. Zhou, Asymmetries in heavy quark pair and dijet production at an EIC, JHEP 08 (2016) 001 [arXiv:1605.07934] [INSPIRE].

[57] G. Billis, M.A. Ebert, J.K.L. Michel and F.J. Tackmann, $A$ toolbox for $q_{T}$ and 0-jettiness subtractions at $N^{3} L O$, arXiv:1909.00811 [INSPIRE].

[58] S. Catani and M. Grazzini, An NNLO subtraction formalism in hadron collisions and its application to Higgs boson production at the LHC, Phys. Rev. Lett. 98 (2007) 222002 [hep-ph/0703012] [INSPIRE].

[59] Y. Li and H.X. Zhu, Bootstrapping rapidity anomalous dimensions for transverse-momentum resummation, Phys. Rev. Lett. 118 (2017) 022004 [arXiv: 1604.01404] [INSPIRE].

[60] T. Gehrmann, T. Luebbert and L.L. Yang, Calculation of the transverse parton distribution functions at next-to-next-to-leading order, JHEP 06 (2014) 155 [arXiv:1403.6451] [INSPIRE].

[61] M.G. Echevarria, I. Scimemi and A. Vladimirov, Transverse momentum dependent fragmentation function at next-to-next-to-leading order, Phys. Rev. D 93 (2016) 011502 [Erratum ibid. D 94 (2016) 099904] [arXiv: 1509.06392] [INSPIRE].

[62] M.G. Echevarria, I. Scimemi and A. Vladimirov, Unpolarized transverse momentum dependent parton distribution and fragmentation functions at next-to-next-to-leading order, JHEP 09 (2016) 004 [arXiv:1604.07869] [INSPIRE].

[63] M.-X. Luo et al., Transverse parton distribution and fragmentation functions at NNLO: the quark case, JHEP 10 (2019) 083 [arXiv:1908.03831] [INSPIRE].

[64] D. Gutierrez-Reyes, S. Leal-Gomez, I. Scimemi and A. Vladimirov, Linearly polarized gluons at next-to-next-to leading order and the Higgs transverse momentum distribution, JHEP 11 (2019) 121 [arXiv:1907.03780] [INSPIRE].

[65] C.W. Bauer, S. Fleming and M.E. Luke, Summing Sudakov logarithms in $B \rightarrow X(s \gamma)$ in effective field theory, Phys. Rev. D 63 (2000) 014006 [hep-ph/0005275] [INSPIRE].

[66] C.W. Bauer, S. Fleming, D. Pirjol and I.W. Stewart, An effective field theory for collinear and soft gluons: heavy to light decays, Phys. Rev. D 63 (2001) 114020 [hep-ph/0011336] [INSPIRE].

[67] C.W. Bauer, D. Pirjol and I.W. Stewart, Soft collinear factorization in effective field theory, Phys. Rev. D 65 (2002) 054022 [hep-ph/0109045] [INSPIRE].

[68] C.W. Bauer et al., Hard scattering factorization from effective field theory, Phys. Rev. D 66 (2002) 014017 [hep-ph/0202088] [INSPIRE]. 
[69] M. Beneke, A.P. Chapovsky, M. Diehl and T. Feldmann, Soft collinear effective theory and heavy to light currents beyond leading power, Nucl. Phys. B 643 (2002) 431 [hep-ph/0206152] [INSPIRE].

[70] P. Nogueira, Automatic Feynman graph generation, J. Comput. Phys. 105 (1993) 279 [INSPIRE].

[71] J.A.M. Vermaseren, New features of FORM, math-ph/0010025 [INSPIRE].

[72] C. Anastasiou and K. Melnikov, Higgs boson production at hadron colliders in NNLO QCD, Nucl. Phys. B 646 (2002) 220 [hep-ph/0207004] [INSPIRE].

[73] A.V. Smirnov, FIRE5: a C++ implementation of Feynman Integral REduction, Comput. Phys. Commun. 189 (2015) 182 [arXiv:1408.2372] [INSPIRE].

[74] R.N. Lee, Presenting LiteRed: a tool for the Loop InTEgrals REDuction, arXiv:1212.2685 [INSPIRE].

[75] K.G. Chetyrkin and F.V. Tkachov, Integration by parts: the algorithm to calculate $\beta$-functions in 4 loops, Nucl. Phys. B 192 (1981) 159 [INSPIRE].

[76] T. Gehrmann and E. Remiddi, Differential equations for two loop four point functions, Nucl. Phys. B 580 (2000) 485 [hep-ph/9912329] [INSPIRE].

[77] J.M. Henn, Multiloop integrals in dimensional regularization made simple, Phys. Rev. Lett. 110 (2013) 251601 [arXiv: 1304.1806] [INSPIRE].

[78] E. Remiddi and J.A.M. Vermaseren, Harmonic polylogarithms, Int. J. Mod. Phys. A 15 (2000) 725 [hep-ph/9905237] [INSPIRE].

[79] D. Maître, HPL, a Mathematica implementation of the harmonic polylogarithms, Comput. Phys. Commun. 174 (2006) 222 [hep-ph/0507152] [INSPIRE].

[80] A.V. Manohar and I.W. Stewart, The zero-bin and mode factorization in quantum field theory, Phys. Rev. D 76 (2007) 074002 [hep-ph/0605001] [INSPIRE].

[81] T. Gehrmann, T. Lubbert and L.L. Yang, Transverse parton distribution functions at next-to-next-to-leading order: the quark-to-quark case, Phys. Rev. Lett. 109 (2012) 242003 [arXiv:1209.0682] [INSPIRE].

[82] M. Stratmann and W. Vogelsang, Next-to-leading order evolution of polarized and unpolarized fragmentation functions, Nucl. Phys. B 496 (1997) 41 [hep-ph/9612250] [INSPIRE].

[83] J. Blumlein, V. Ravindran and W.L. van Neerven, On the Drell-Levy-Yan relation to $O\left(\alpha_{s}^{2}\right)$, Nucl. Phys. B 586 (2000) 349 [hep-ph/0004172] [INSPIRE].

[84] A. Mitov, S. Moch and A. Vogt, Next-to-next-to-leading order evolution of non-singlet fragmentation functions, Phys. Lett. B 638 (2006) 61 [hep-ph/0604053] [INSPIRE].

[85] S. Moch and A. Vogt, On third-order timelike splitting functions and top-mediated Higgs decay into hadrons, Phys. Lett. B 659 (2008) 290 [arXiv:0709.3899] [INSPIRE].

[86] A.A. Almasy, S. Moch and A. Vogt, On the next-to-next-to-leading order evolution of flavour-singlet fragmentation functions, Nucl. Phys. B 854 (2012) 133 [arXiv:1107.2263] [INSPIRE].

[87] M.-X. Luo, V. Shtabovenko, T.-Z. Yang and H.X. Zhu, Analytic next-to-leading order calculation of energy-energy correlation in gluon-initiated Higgs decays, JHEP 06 (2019) 037 [arXiv: 1903.07277] [INSPIRE]. 
[88] L.J. Dixon, I. Moult and H.X. Zhu, Collinear limit of the energy-energy correlator, Phys. Rev. D 100 (2019) 014009 [arXiv: 1905.01310] [INSPIRE].

[89] T.Z. Yang, unpublished work.

[90] M. Kologlu, P. Kravchuk, D. Simmons-Duffin and A. Zhiboedov, The light-ray OPE and conformal colliders, arXiv:1905.01311 [INSPIRE].

[91] G.P. Korchemsky, Energy correlations in the end-point region, arXiv:1905.01444 [INSPIRE].

[92] G.P. Korchemsky and A.V. Radyushkin, Renormalization of the Wilson loops beyond the leading order, Nucl. Phys. B 283 (1987) 342 [INSPIRE].

[93] I.A. Korchemskaya and G.P. Korchemsky, On lightlike Wilson loops, Phys. Lett. B 287 (1992) 169 [INSPIRE].

[94] A.A. Vladimirov, Correspondence between soft and rapidity anomalous dimensions, Phys. Rev. Lett. 118 (2017) 062001 [arXiv: 1610.05791] [INSPIRE].

[95] T. Gehrmann, T. Huber and D. Maître, Two-loop quark and gluon form-factors in dimensional regularisation, Phys. Lett. B 622 (2005) 295 [hep-ph/0507061] [INSPIRE].

[96] T. Becher and M. Neubert, On the structure of infrared singularities of gauge-theory amplitudes, JHEP 06 (2009) 081 [Erratum ibid. 11 (2013) 024] [arXiv:0903.1126] [INSPIRE].

[97] Y. Li, A. von Manteuffel, R.M. Schabinger and H.X. Zhu, Soft-virtual corrections to Higgs production at $N^{3} L O$, Phys. Rev. D 91 (2015) 036008 [arXiv: 1412.2771] [INSPIRE].

[98] D.J. Gross and F. Wilczek, Ultraviolet behavior of nonabelian gauge theories, Phys. Rev. Lett. 30 (1973) 1343 [INSPIRE].

[99] H.D. Politzer, Reliable perturbative results for strong interactions?, Phys. Rev. Lett. 30 (1973) 1346 [InSPIRE].

[100] W.E. Caswell, Asymptotic behavior of nonabelian gauge theories to two loop order, Phys. Rev. Lett. 33 (1974) 244 [INSPIRE].

[101] D.R.T. Jones, Two loop diagrams in Yang-Mills theory, Nucl. Phys. B 75 (1974) 531 [INSPIRE].

[102] E. Egorian and O.V. Tarasov, Two loop renormalization of the QCD in an arbitrary gauge, Teor. Mat. Fiz. 41 (1979) 26 [INSPIRE].

[103] G. Altarelli and G. Parisi, Asymptotic freedom in parton language, Nucl. Phys. B 126 (1977) 298 [inSPIRE].

[104] W. Furmanski and R. Petronzio, Singlet parton densities beyond leading order, Phys. Lett. B 97 (1980) 437.

[105] G. Curci, W. Furmanski and R. Petronzio, Evolution of parton densities beyond leading order: the nonsinglet case, Nucl. Phys. B 175 (1980) 27 [INSPIRE]. 